\title{
Retinoic Acid Promotes Apoptosis and Differentiation in Photoreceptors by Activating the P38 MAP Kinase Pathway
}

\author{
Pablo De Genaro, ${ }^{1}$ M. Victoria Simón, ${ }^{1,2}$ Nora P. Rotstein, ${ }^{1,2}$ and Luis E. Politi ${ }^{1,2}$ \\ ${ }^{1}$ Instituto de Investigaciones Bioquímicas de Bahía Blanca (INIBIBB), Buenos Aires, Argentina \\ ${ }^{2}$ Universidad Nacional del Sur, Bahía Blanca, Buenos Aires, Argentina
}

Correspondence: Luis E. Politi INIBIBB - Camino La Carrindanga Km 7 - 8000 Bahía Blanca, Buenos Aires, Argentina;

inpoliti@criba.edu.ar.

Submitted: September 28, 2012

Accepted: March 26, 2013

Citation: De Genaro P, Simón MV, Rotstein NP, Politi LE. Retinoic acid promotes apoptosis and differentiation in photoreceptors by activating the P38 MAP kinase pathway. Invest Ophthalmol Vis Sci. 2013;54:31433156. DOI:10.1167/iovs.12-11049

\begin{abstract}
Purpose. Retinoic acid (RA) has a critical role during development of the retina. We investigated RA effects on photoreceptor apoptosis and differentiation, and the intracellular pathways involved.
\end{abstract}

Methods. Rat retinal neuronal cultures were supplemented with RA with or without docosahexaenoic acid (DHA), a photoreceptor survival factor, and photoreceptor apoptosis and differentiation were evaluated at different times of development. To investigate the intracellular pathways activated by RA, the levels of phosphorylated (P) ERK and P-p38 in cultures with or without RA, and the effect of pretreatment with SB203580, a p38 specific inhibitor, on apoptosis and differentiation were evaluated.

Results. RA addition at day 0 , when cells still were proliferating, selectively increased apoptosis in photoreceptors, whereas addition at day 2 no longer caused cell death. RA stimulated opsin and peripherin expression, and neurite outgrowth regardless of the time of development. Addition of RA at day 0, but not at day 2, rapidly increased P-p38 levels, but did not affect P-ERK levels. p38 inhibition completely prevented RA-induced apoptosis, and partially decreased differentiation. DHA prevented apoptosis and additively increased differentiation, without affecting RA activation of $\mathrm{p} 38$.

Conclusions. Our results show that RA activation of the p38 intracellular pathway was essential for its early induction of apoptosis and partially responsible for promoting differentiation. DHA prevention of this apoptosis suggests that RA effects during early development must be counterbalanced by survival factors to prevent photoreceptor death, in an interplay that might help to establish the final number of photoreceptors.

Keywords: photoreceptors, retinoid, apoptosis

\begin{abstract}
A poptosis is an integral part of development. Of the huge Asurplus of cells generated during development of the nervous system, those that fail to establish adequate connections and get survival signals die through apoptosis, which contributes to sculpting a functional system. In the retina, after synaptogenesis, photoreceptors need several survival and trophic factors to prevent this apoptosis and continue their development. ${ }^{1,2}$ Similarly, in cultures lacking trophic factors, photoreceptors start to degenerate and die after 4 days in vitro, following an apoptotic pathway. ${ }^{3}$ Photoreceptor trophic factors, such as glial derived neurotrophic factor (GDNF), basic fibroblast growth factor (FGFb), ciliary neurotrophic factor (CNTF), and docosahexaenoic acid (DHA) are required to promote photoreceptor survival in vivo and in vitro. ${ }^{3-8}$

Another set of signaling molecules, such as taurine and alltrans-retinoic acid (atRA), act earlier in development. ${ }^{9,10} \mathrm{RA}$ is a crucial inducer of differentiation in many cell types, among them retina neurons. ${ }^{11-14}$ It has a critical role during the development of photoreceptors in vivo and in vitro, ${ }^{10,15}$ determining cell fate and promoting opsin expression. ${ }^{16,17}$

Photoreceptor trophic factors, such as FGFb, GDNF, and DHA, have their pro-survival actions coupled to an advancement of differentiation, which seems reasonable for establishing a mature, functional system. ${ }^{3,6,8,18}$ This appears not to be the case for RA, which triggers apoptosis of NT2 cells, a human
\end{abstract}

teratocarcinoma cell line, when inducing their differentiation to a neuronal phenotype. ${ }^{19,20}$ In mouse retinal explants 9-cis-RA induces photoreceptor apoptosis, simultaneously increasing opsin expression. ${ }^{17}$

Though known to modulate numerous signaling pathways in different cell types, the intracellular pathways activated by RA to achieve its effects on photoreceptors remain to be established. To investigate them, we have used pure neuronal cultures from neonatal rat retinas, which initially present retina progenitors that later differentiate as amacrine and photoreceptor cells. This allows us to evaluate the effects of RA on photoreceptor differentiation and apoptosis. Our results showed that RA rapidly advanced the differentiation of photoreceptors at different times of development, but only induced their early apoptosis if added when cells still were cycling. They also evidenced that RA effects on photoreceptor apoptosis depended on the activation of the p38 MAP kinase (p38 MAPK) signaling pathway, whereas differentiation involved this mechanism together with other signaling pathways. DHA, a photoreceptor survival molecule, prevented RAinduced apoptosis and had an additive effect on differentiation. Our results suggested that a fine balance between the levels of RA and those of survival molecules is indispensable during development to promote differentiation and simultaneously preserve the adequate number of photoreceptors in the retina. 


\section{Materials ANd Methods}

Albino Wistar rats bred in our own colony were used in all experiments. All proceedings concerning animal use were done in accordance with the ARVO Statement for the Use of Animals in Ophthalmic and Vision Research, and the guidelines published in the NIH Guide for the Care and Use of Laboratory Animals (available in the public domain at http://grants.nih.gov/grants/olaw/Guide-for-the-Care-and-Useof-Laboratory-Animals.pdf). Plastic culture dishes (CellStar) were from Greiner Bio-One (Frickenhausen, Germany). Dulbecco's modified Eagle's medium (DMEM; Gibco) was purchased from Life Technologies (Grand Island, NY). All-trans retinoic acid (98\% purity by HPLC), poly-L-ornithine, trypsin, trypsin inhibitor, transferrin, hydrocortisone, putrescine, insulin, CDP-choline, CDP-ethanolamine, gentamicin, 4,6-diamidino-2-phenylindole (DAPI), paraformaldehyde, (4-[4'-fluo-

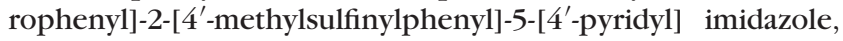
SB203580), DHA, monoclonal anti-acetylated tubulin and anti-syntaxin (HPC-1) antibodies were from Sigma-Aldrich (St. Louis, MO). Polyclonal antibodies against $P-p 38$, total $p 38, P$ Erk1-2, and total Erk1-2 were from Cell Signaling Technologies (Beverly, MA). Monoclonal antibodies against rhodopsin (Rho4D2) and peripherin (clone p3b6) were generously supplied by Robert Molday (University of British Columbia, Vancouver, BC, Canada). Polyclonal anti-Crx antibody was a generous gift of Dr Cheryl Craft (University of Southern California, Los Angeles, CA). Polyclonal anti-Pax6 antibody was a kind gift of Dr GS Mastick (University of Michigan, Ann Arbor, MI). Cy2 and Cy3 conjugated goat anti-rabbit secondary antibodies were from Jackson ImmunoResearch (West Grove, PA).

Monoclonal antibodies anti-actin, nestin, p2 $7^{\mathrm{kip} 1}$, and secondary antibodies used for Western Blot analysis, goat anti-mouse IgG-horseradish peroxidase (HRP), and goat antirabbit IgG-HRP, were from Santa Cruz Biotechnology, Inc. (Santa Cruz, CA). Tyramide Signal Amplification kit (TSA) was from DuPont NEN (Wilmington, Denmark). Monoclonal antibody against BrdU (clone G3G4) was from DSHB (developed under the auspices of the NICHD and maintained by the University of Iowa, Department of Biological Sciences, Iowa City, IA). MitoTracker Red CMXRos and terminal deoxynucleotidyl transferase, recombinant 5-bromo-2-deoxyuridine-5-triphosphate (BrdUTP), and terminal deoxy-nucleotidyl transferase (TdT) buffer were from Molecular Probes (Eugene, OR). Cell-permeant pan caspase inhibitor, carbobenzoxy-valylalanyl-aspartyl-(O-methyl)-fluoromethylketone (Z-VAD-FMK) was from Promega (Madison, WI). Polyvinylidene difluoride (PVDF) membranes were Immobilon P (Millipore, Billerica, MA). Solvents were HPLC grade, and all other reagents were analytical grade.

\section{Neuronal Cultures}

Pure neuronal cultures were obtained from 0- or 2-day-old (PNO-2) rat retinas as described previously. ${ }^{3}$ In brief, retinas were dissected and dissociated under mechanical and chemical digestion with $0.25 \%$ trypsin. Cells then were resuspended and seeded in a chemically defined medium, ${ }^{3,21}$ in dishes pretreated sequentially with poly-L-Ornithine and Schwannoma conditioned medium. ${ }^{22}$ Cultures were incubated at $36^{\circ} \mathrm{C}$ in a $5 \% \mathrm{CO}_{2}$ atmosphere. Retinal progenitors differentiated mainly into amacrine neurons and rod photoreceptors, which amounted to approximately $30 \%$ and $70 \%$ of total cells, respectively. Occasionally, a few glial cells were observed (less than $1 \%$ of total cells), which did not proliferate due to the high adhesive substrata, low cell density, and lack of serum. Neuronal cell types were identified by their morphology, using phase- contrast microscopy and by immunocytochemistry, using the monoclonal antibodies anti-syntaxin (HPC-1) and Rho4D2, for amacrine and photoreceptor neurons, respectively. ${ }^{23-25}$ Photoreceptors have a small round cell body (3-5 $\mu \mathrm{m}$ in diameter) with a single neurite, which usually ends in a conspicuous synaptic "spherule"; sometimes they display a connecting cilium at the opposite end, but they fail to develop their characteristic outer segments; opsin is distributed diffusely over their cell body, which usually is darker than that of amacrine neurons. To be identified as photoreceptors, the cells had to display at least three of the above described criteria. Amacrine neurons are larger than photoreceptors (7-20 $\mu \mathrm{m}$ in diameter) and have multiple neurites. Almost all of them show HPC-1 immunoreactivity starting at early stages of development, which is retained even after undergoing degenerative changes that alter their morphologic appearance. ${ }^{26}$

\section{Addition of RA}

Stock solutions of all-trans RA (1 $\mathrm{mM}$ in absolute ethanol, $\mathrm{EtOH})$ were prepared, maintained in the dark at $-70^{\circ} \mathrm{C}$, and diluted further in DMEM at the time of supplementation; aliquots were added under dim light to reduce RA degradation. RA was added 1 hour after seeding the cells, or at day 2. To establish the effect of different concentrations of RA on differentiation and apoptosis, dilutions ranging from $10^{-6}$ to $10^{-9} \mathrm{M}$ were used. In every case, EtOH concentration was below $0.01 \%$ (final concentration in culture). The same EtOH concentration was added to control cultures.

\section{Addition of DHA}

DHA (6.7 $\mu \mathrm{m}$ final concentration in culture) was added at day 0 , in a complex with BSA. ${ }^{3}$ A BSA solution was added to control cultures.

\section{Evaluation of Proliferation and Cell Fate}

To assess proliferation, cultures from PNO retinas were treated with or without RA at day 0 , incubated for 4 hours with $30 \mu \mathrm{M}$ BrdU (final concentration in culture), and fixed after 12 or 48 hours in vitro with $4 \%$ paraformaldehyde. BrdU uptake was determined using a monoclonal antibody against BrdU.

To determine the number of proliferating progenitors we evaluated (1) the expression of the neuroectoderm marker, nestin; (2) the expression of the cell cycle blocker, p2 $7^{\mathrm{kip} 1}$; and (3) the percentage of cells expressing Pax 6 during the first 48 hours after RA addition. Though Pax6 is expressed in every mitotic retina progenitor, ${ }^{27}$ its expression only remains in postmitotic amacrine neurons. To evaluate proliferation exclusively, we excluded cells that coexpressed HPC-1, an amacrine cell marker, and Pax6. To analyze RA effect on cell fate, we determined the amount of cells expressing Crx, a photoreceptor marker, and HPC-1 after 24 or 48 hours in vitro, using specific monoclonal antibodies.

\section{Activation of the p38 MAPK Pathway}

To establish whether the activation of the p38 MAPK pathway was involved in RA effects on apoptosis and differentiation, SB203580, a specific, cell permeant inhibitor of p38, was used. A stock solution was prepared in DMSO and diluted in DMEM before addition to the cultures $(100 \mathrm{nM}$, final concentration in culture). To analyze whether RA promoted the phosphorylation of $\mathrm{p} 38$, cells were treated with RA or vehicle at days 0 and 2 , and either fixed or scraped for immunocytochemical or Western blot analyses, using a polyclonal antibody against phosphorylated (P)-p38. 


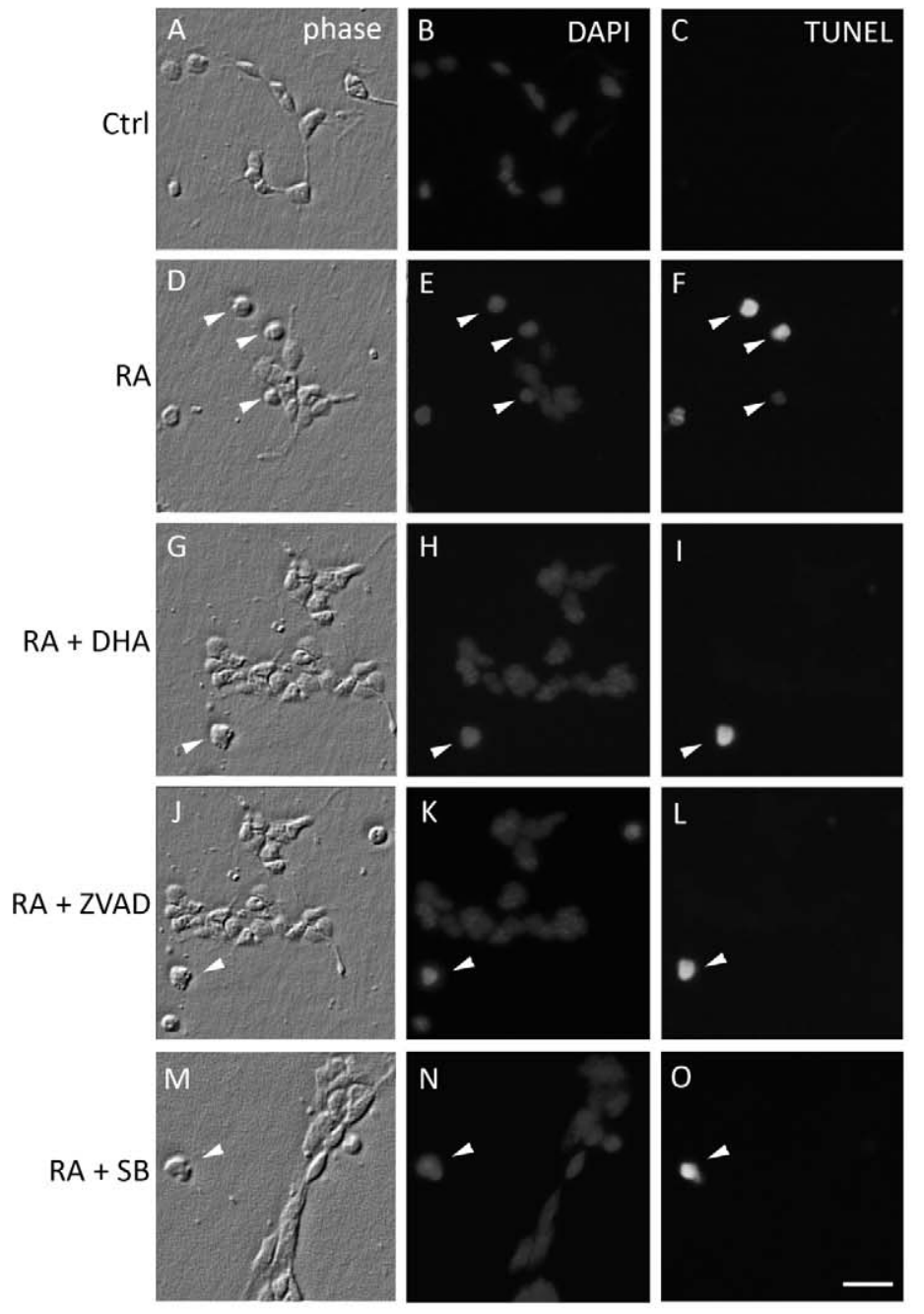

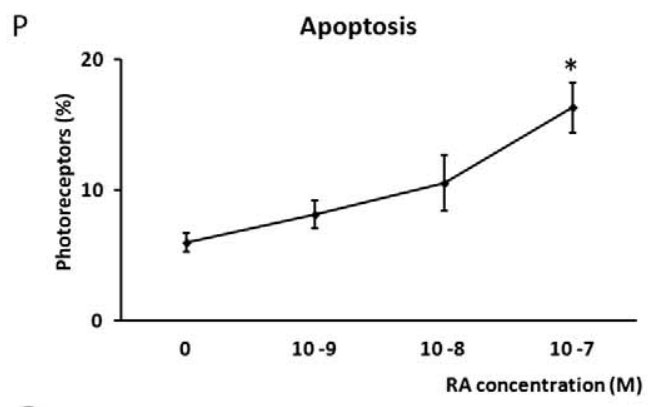

Q

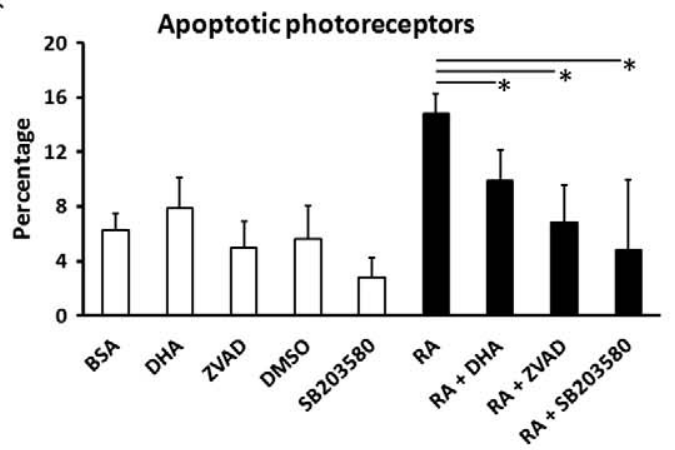

$\mathrm{R}$

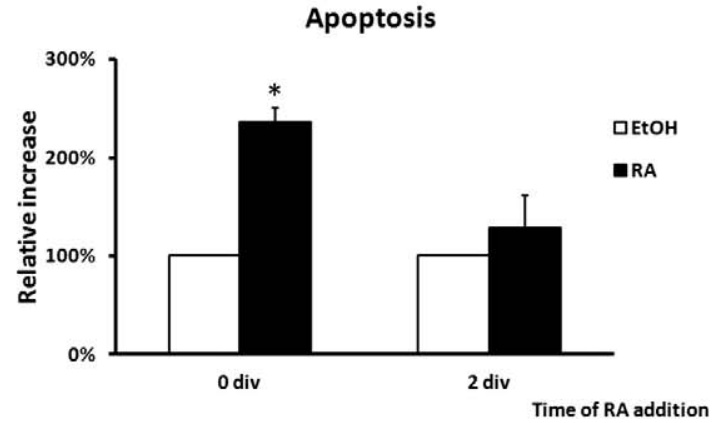

FiguRE 1. Effect of RA on cell survival. Neuronal cultures were treated with vehicle (EtOH, control, [A-C]), RA (D-F), RA and DHA (in a BSA solution, [G-I]), the pan-caspase inhibitor Z-VAD-FMK (ZVAD, [J-L]), or the p38MAPK specific inhibitor SB203580 (SB, [M-O]) at day 0, and apoptosis was evaluated at day 3. Phase contrast (A, D, G, J, M) and fluorescence $(\mathbf{B}, \mathbf{C}, \mathbf{E}, \mathbf{F}, \mathbf{H}, \mathbf{I}, \mathbf{K}, \mathbf{L}, \mathbf{N}, \mathbf{O})$ photomicrographs show that RA induced apoptosis in photoreceptors, as evidenced by the increase in the amount of pyknotic nuclei (nuclei with condensed and bright DAPI staining, arrowheads) and TUNEL-positive cells, as compared to control cultures. Pretreatment with either DHA (G-I), Z-VAD (J-L), or SB (M-O) reduced RA-induced apoptosis. The effect of increasing concentrations of RA on photoreceptor apoptosis is shown in (P). Quantification of the percentage of apoptotic photoreceptors (Q), by analyzing nuclei integrity with DAPI, showed a significant increase in apoptosis in RA-treated cultures, and the protective effect of DHA, and the p38 and pan-caspase inhibitors. The effect of RA addition at days 0 and 2 in culture on photoreceptor apoptosis is compared in $(\mathbf{R})$; RA only induced apoptosis when added at day $0 .{ }^{*} P<0.05$. Scale bar: $15 \mu \mathrm{m}$.

\section{Activation of the ERK/MAPK Pathway}

To investigate whether RA modulated the ERK/MAPK pathway, cells were treated with RA or vehicle at day 0 , and either fixed or scraped for immunocytochemical or Western Blot analyses. P- and total ERK were determined using polyclonal antibodies.

\section{Immunocytochemical Methods}

Cultures were fixed with $4 \%$ paraformaldehyde in PBS, for at least 30 minutes at room temperature, followed by permeation with Triton X-100 (0.1\% in PBS). For immunocytochemistry, Cy2 and Cy3-conjugated goat anti-mouse or anti-rabbit were used as secondary antibodies. TSA occasionally was used to improve visualization. Controls were performed by omitting either the primary or the secondary antibodies. Cultures were analyzed by phase contrast and fluorescence microscopy.

\section{Evaluation of Photoreceptor Differentiation}

To evaluate whether RA advanced the differentiation of photoreceptors, we determined the amount of cells expressing opsin, the visual pigment, and peripherin, a disk membrane protein, and the amount of photoreceptors having either opsin- or peripherin-positive apical processes, using Rho4D2 and anti-peripherin monoclonal antibodies, respectively.

\section{Analysis of Neurite Outgrowth}

Neurites were labeled with an anti $\alpha$-acetylated tubulin antibody. To distinguish between photoreceptor and amacrine cell neurites, we determined the number of neurons coexpressing $\alpha$-acetylated tubulin with either Crx or HPC-1, respectively. To assess neurite outgrowth we evaluated the amount of neurons bearing long neurites, defined as those whose length exceeded 3 and 4 cell body diameters, for photoreceptors and amacrine cells, respectively. 

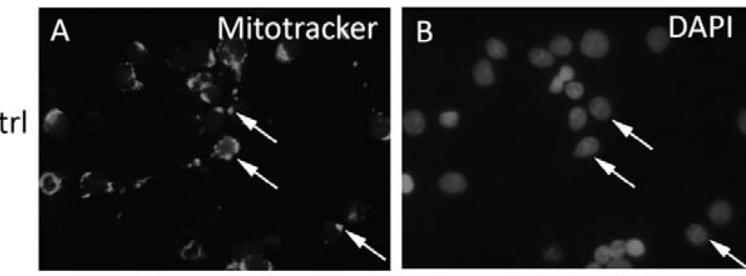

RA
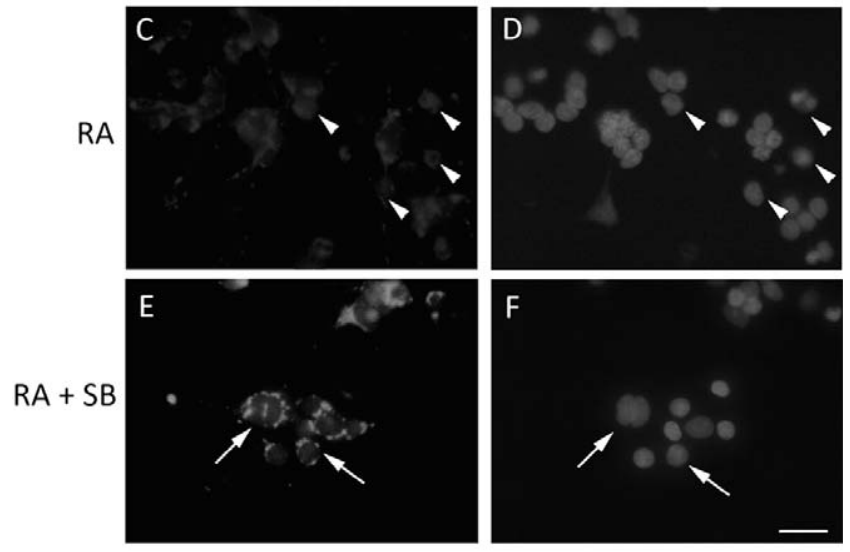

G Photoreceptors having active mitochondria

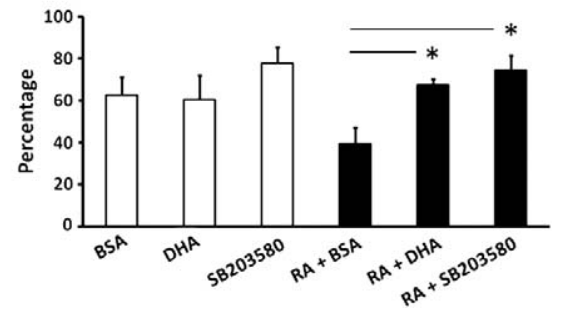

Figure 2. Effect of RA on mitochondrial membrane potential. Cultures were treated at day 0 with or without RA, and mitochondrial membrane potential (A, C, E) and nuclei integrity $(\mathbf{B}, \mathbf{D}, \mathbf{F})$ were evaluated after 3 days in vitro, using MitoTracker and DAPI, respectively. Fluorescence photomicrographs show control cultures (A, B) had more photoreceptors preserving mitochondrial membrane potential (bright localized fluorescence in $[\mathbf{A}]$, arrows) than RA-treated cultures $(\mathbf{C}, \mathbf{D})$, which showed unlocalized dim fluorescence ([C], arrowheads). Treatment with the p38 inhibitor SB203580 before RA addition (E, F) prevented RA-induced mitochondrial membrane depolarization ([E], arrows). The percentage of photoreceptors preserving mitochondrial membrane potential is shown in (G); note that RA significantly decreased the amount of photoreceptors having active mitochondria, while pretreatment with DHA and SB203580 prevented this decrease. ${ }^{*} P<0.05$. Scale bar: $15 \mu \mathrm{m}$.

\section{Evaluation of Apoptosis}

Apoptosis was determined by evaluation of nuclei integrity and by TUNEL assay. ${ }^{28}$ Nuclear integrity was evaluated by staining cell nuclei with DAPI; neurons were considered apoptotic when they showed either fragmented or condensed (pyknotic) nuclei. The number of apoptotic photoreceptors and amacrine cells was determined in cultures double-labeled with DAPI and Rho4D2 or HPC-1, respectively.

To investigate whether RA induced apoptosis through a caspase-dependent pathway, day 0 cultures were treated with a cell-permeant, pan-caspase inhibitor, Z-VAD-FMK, in DMEM (20 $\mu \mathrm{M}$, final medium concentration) and one hour later with or without RA. Apoptosis was determined at day 3, by evaluating nuclei integrity.
TABle 1. Effect Of RA on Amacrine Cell Survival

\begin{tabular}{lcc}
\hline Treatment & Apoptotic Cells, $\%$ & Active Mitochondria, \% \\
\hline EtOH & $1.5 \pm 0.3$ & $93.8 \pm 2.8$ \\
RA & $1.7 \pm 0.1$ & $94.8 \pm 1.5$ \\
\hline
\end{tabular}

The percentage of apoptotic amacrine neurons and of amacrine neurons preserving their mitochondrial membrane potential at day 3 was determined by immunocytochemistry using DAPI and Mitotracker, respectively.

\section{Evaluation of Mitochondrial Membrane Potential}

Changes in mitochondrial membrane potential were analyzed by incubating cultures for 20 minutes before fixation with the fluorescent probe MitoTracker Red $(0.1 \mathrm{mg} / \mathrm{mL})$, which labels mitochondria retaining their membrane potential with a bright red fluorescence.

\section{Microscopy}

Cultures were analyzed by phase contrast and epifluorescence microscopy, using a Nikon Eclipse E600 microscope with a C-C Phase Contrast Turret Condenser and a Y-FL Epi-Fluorescence Attachment (Nikon Instruments, Inc., Mellville, NY), and a laser scanning confocal microscope (Leica DMIRE2; Leica Microsystems, Wetzler, Germany) with a 63X water objective; images were collected and processed with LCS software (Leica Microsystems) and Photoshop 8.0 (Adobe Systems, San Jose, CA).

\section{Western Blotting}

Western blot was performed to investigate protein expression. ${ }^{29}$ Proteins were quantified (DC, Bio-Rad; Hercules, CA), subjected to one-dimensional electrophoresis, ${ }^{30}$ transferred to PVDF membranes, and visualized with enhanced chemiluminescence (ECL Plus Western Blotting Detection Reagents - RPN 2132; GE Healthcare, Silver Spring, MD). Images were obtained by scanning at $600 \mathrm{dpi}$ and bands were quantified with ImageJ software (NIH).

\section{Statistical Analysis}

For cytochemical studies, 10 fields per sample, randomly chosen, were analyzed in each case. Each value represented the average of at least three experiments, with three to four dishes for each condition \pm SD. Statistical significance was determined by Student's 2-tailed $t$-test.

\section{Results}

\section{RA-Induced Photoreceptor Apoptosis}

We first evaluated the effect of RA addition at day 0 on cell death. By day 3, cultures lacking RA showed virtually no TUNEL-positive cells, and approximately $6 \%$ of photoreceptors had fragmented or pyknotic nuclei (Figs. 1A-C, 1P, 1Q). Addition of $10^{-7} \mathrm{M} \mathrm{RA}$ increased this percentage to approximately $16 \%$. Since $10^{-9} \mathrm{M}$ and $10^{-8} \mathrm{M}$ RA induced a slight, though nonsignificant, increase in apoptosis (Fig. 1P) and higher $\left(10^{-6} \mathrm{M}\right) \mathrm{RA}$ concentrations provoked morphologic changes (abnormal cell morphology and neurite fragmentation and cell detachment, not shown), $10^{-7} \mathrm{M}$ RA was used in subsequent experiments.

To investigate whether time of development in vitro influenced RA-induced apoptosis of photoreceptors, RA was added at day 0 or 2 . At day 3 , controls showed very few 

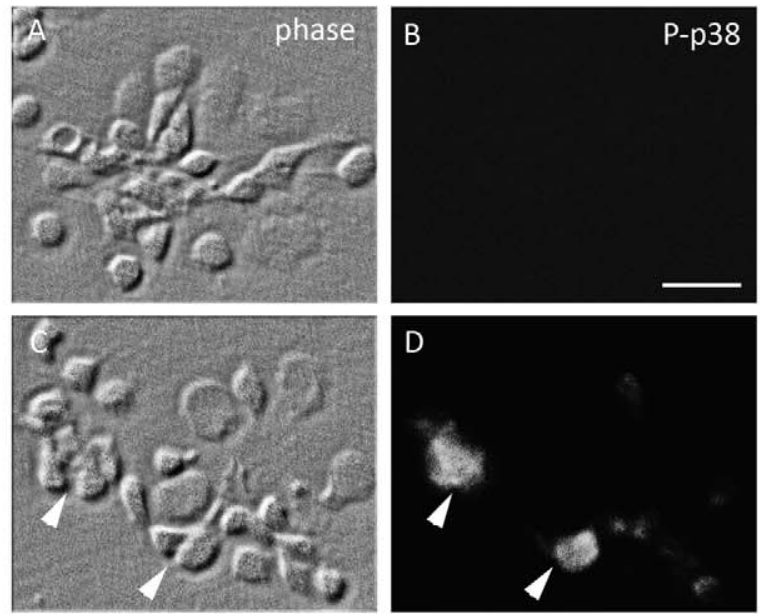

$E$
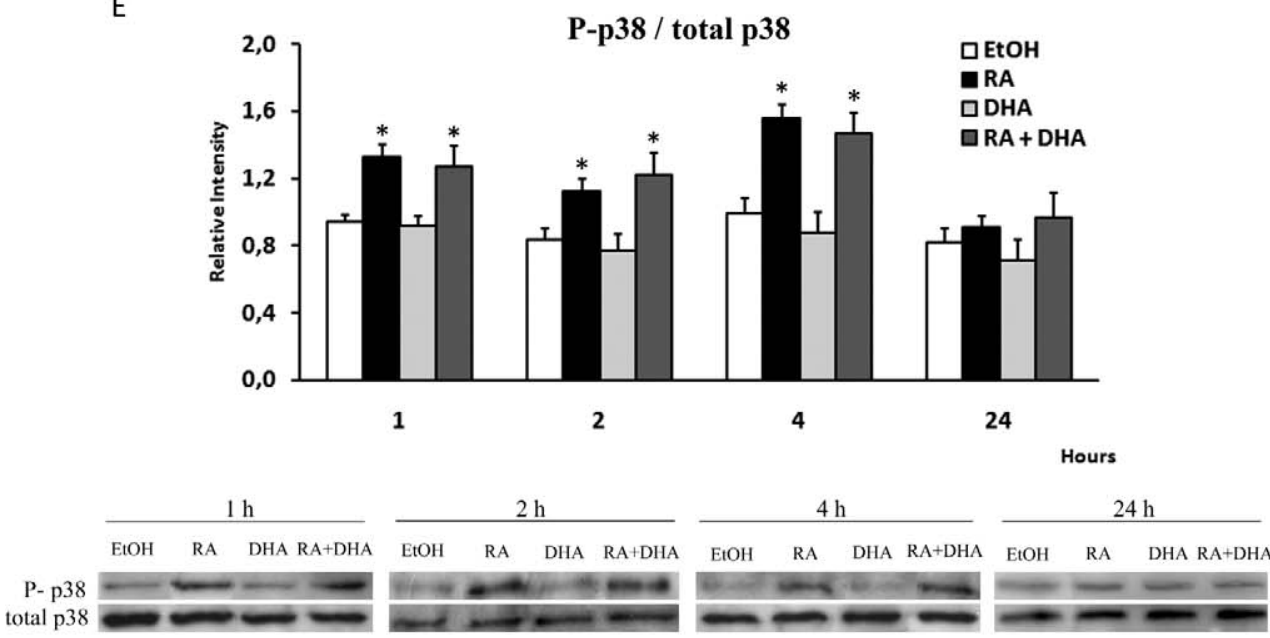

$\mathrm{F}$
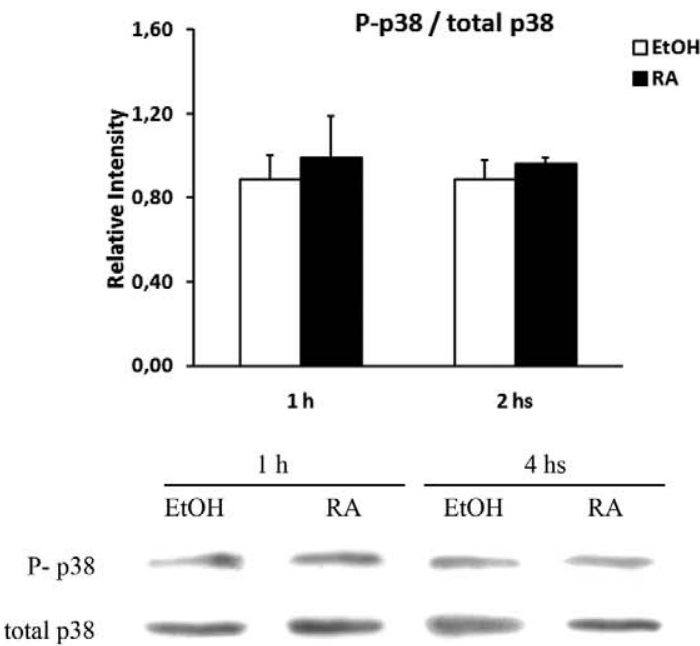

Figure 3. Activation of p38 MAPK by RA. Phase contrast (A, C) and fluorescence (B, D) photomicrographs show expression of P-p38 in control (A B) and RA-treated cultures (C, D) analyzed by immunocytochemistry at day 0. RA markedly increased P-p38 levels (note P-p38 labeled cells in [D], almost absent in $[\mathbf{B}]$ ), particularly in photoreceptors (arrowheads in $[\mathbf{D}]$ ). Western blots (lower panel) and relative changes in P-p38 expression (upper panel) at different times of incubation with RA, DHA, RA plus DHA, or EtOH added at day 0 are shown in (E). RA induced a rapid increase in P-p38 levels between 1 and 4 hours after treatment, which decreased to control levels after 24 hours. Note that DHA addition neither elicited a p38 response nor blocked the RA-induced increase in P-p38, thus suggesting that DHA activated a different signaling pathway. Western blots (lower panel) and relative changes in P-p38 expression (upper panel) in cultures treated without or with RA, added at day 2 are shown in (F); note that at this time point RA no longer induced p38 activation. ${ }^{*} P<0.05$. Scale bar: $15 \mu \mathrm{m}$. 

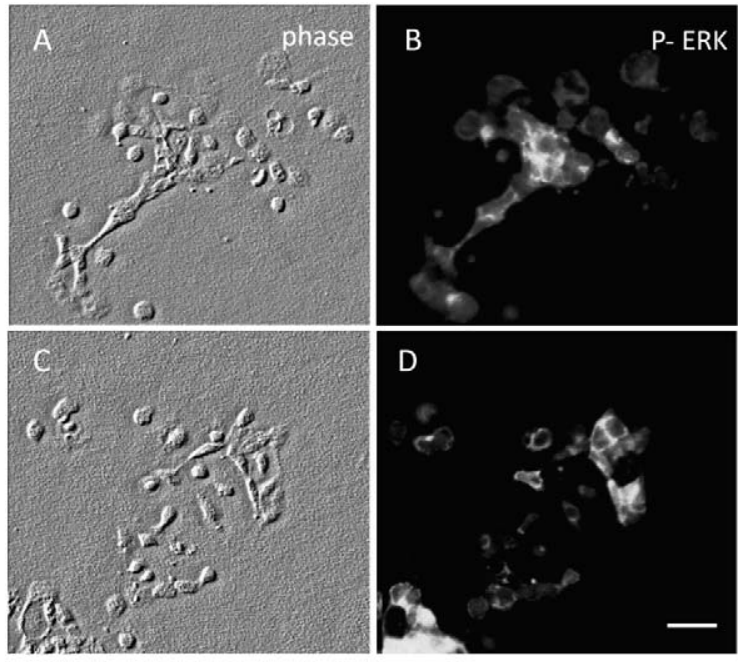

$E$ $\frac{1 \mathrm{~h}}{\mathrm{EtOH} \text { RA }} \frac{2 \mathrm{hs}}{\mathrm{EtOH} \text { RA }} \frac{4 \mathrm{hs}}{\mathrm{EtOH}} \frac{24 \mathrm{hs}}{\mathrm{EtOH}}$

P-ERK

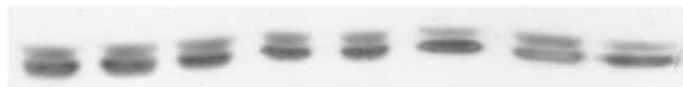

total ERK

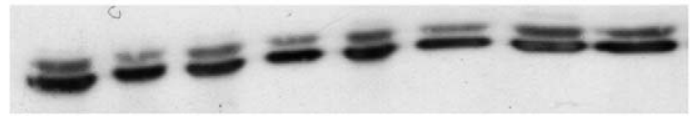

Figure 4. Effect of RA on ERK/MAPK signaling. Day 0 control (A, B) and RA-treated cultures $(\mathbf{C}, \mathbf{D})$ were incubated for different times after RA addition and then cells were fixed for immunocytochemical analysis, using a polyclonal phosphorylated P-ERK1-2 antibody, or scraped for Western blot analysis, using anti P-ERK or anti total ERK antibodies. Phase contrast (A, C) and fluorescence (B, D) photomicrographs show P-ERK labeled cells in control (A, B) and RA-treated (C, D) cultures, 24 hours after RA supplementation; note that RA did not affect P-ERK labeling (compare [A-D]). Western blots (E) of P-ERK after $1,2,4$, and 24 hours of RA treatment evidenced no significant differences between controls and RA-supplemented cultures. Scale bar: $15 \mu \mathrm{m}$.

apoptotic cells, as photoreceptor degeneration had not yet started $^{4}$ (Figs. 1A-C). RA addition at day 0, when many photoreceptor precursors still are active in the cell cycle, ${ }^{27,31}$ induced a premature onset of apoptosis; TUNEL-positive cells clearly were evident (Figs. 1D-F) and the percentage of photoreceptors showing pyknotic nuclei increased from approximately $6 \%$ in controls to $14.8 \%$ after RA treatment (Fig. 1Q).

In contrast, RA addition by day 2 , when most cells have exited the cell cycle, ${ }^{27}$ did not increase photoreceptor apoptosis. By day 4, the percentage of apoptotic photoreceptors in RA-treated cultures showed no significant differences compared to controls (Fig. 1R).

Pretreatment with Z-VAD-FMK, a pan-caspase inhibitor, before RA supplementation completely blocked RA-induced apoptosis (Figs. 1J-L, 1Q). This apoptotic pathway involved mitochondrial membrane depolarization. At day 1, approximately $70 \%$ of photoreceptors preserved their mitochondrial membrane potential, with or without RA (not shown). By day 3, RA decreased the amount of photoreceptors preserving mitochondrial polarization (Figs. 2C, 2D, 2G). While approximately $60 \%$ of photoreceptors retained their mitochondrial membrane potential in controls (Figs. 2A, 2B, 2G), this percentage decreased to $39.5 \%$ in RA-treated cultures (Fig. 2G).

\section{RA-Induced Cell Death was Selective for Photoreceptors}

RA selectively induced photoreceptor apoptosis. Apoptotic amacrine cells in 3-day cultures were approximately $1.5 \%$, with or without RA, and showed no statistical differences at later times in vitro (Table 1). Consistently, the percentages of amacrine neurons preserving mitochondrial membrane potential were very similar in control and RA-treated cultures (Table $1)$.

\section{RA Activated the p38 Signaling Pathway in Photoreceptors}

RA has been shown to activate p38 MAPK, a stress-responsive serine kinase that is linked to regulation of apoptosis in several cell types. ${ }^{32-37}$ To investigate whether RA activated p38 MAPK in photoreceptors, we evaluated the levels of P-p38, its active form. RA addition at day 0 visibly increased P-p38 expression, compared to controls, mainly in photoreceptors (arrows in Figs. 3C, 3D). The increase was very rapid; Western Blot analysis revealed higher levels of P-p38, compared to controls, 1 hour after adding RA (Fig. 3E), and increments of approximately $40 \%$ and $30 \%$ in the P-p38/p38 ratio 2 and 4 hours after RA addition (Fig. 3E). This increase was transient, as 24 hours after RA addition P-p38 levels were similar to those in controls (Fig. 3E). Noteworthy, RA addition at day 2 in vitro, which did not induce apoptosis, did not increase P-p38 levels (Fig. 3F).

Pretreatment with the p38 inhibitor SB203580 almost completely prevented RA-induced apoptosis; the amount of TUNEL-positive cells was markedly reduced (Figs. 1M-O) and apoptotic photoreceptors decreased from 14.8\% in RA-treated cultures to approximately 5\% in cultures with SB203580 and RA (Fig. 1Q). Mitochondrial membrane potential, which was preserved in 80\% of photoreceptors in cultures with SB203580 and lacking RA, was reduced to $39.5 \%$ with RA addition (Fig. $2 \mathrm{G})$, but almost completely preserved in cultures pretreated with SB203580 before RA addition (Figs. 2E, 2F). These results imply that RA activation of the p38MAPK pathway is crucial to induce photoreceptor apoptosis.

Since DHA and FGF activate the ERK/MAPK pathway to promote photoreceptor survival, ${ }^{6,38}$ we investigated whether RA modulated this pathway to trigger apoptosis. No significant differences were observed in P-ERK labeling between controls and RA-treated cultures at day 1 (Figs. 4A-D). Western Blot analysis evidenced no increase either in P-ERK or in the P-ERK/ ERK ratio, compared to controls, after 1, 2, 4, and 24 hours of RA addition to day 0 cultures (Fig. 4E).

\section{DHA Prevented RA-Induced Apoptosis}

We found intriguing that RA, which is essential for retina development, concurrently triggered cell death in developing photoreceptor progenitors. This implied that survival molecules should be present at the same time of development to counteract RA pro-apoptotic effect. Since photoreceptors depend on several trophic and survival molecules to prevent apoptosis, including DHA, ${ }^{4,38-42}$ we evaluated RA effect on photoreceptor apoptosis in cultures without or with DHA. By day 3, RA significantly increased photoreceptor apoptosis, compared to controls (Figs. 1B, 1C, 1E, 1F), but supplementation with DHA before RA addition completely prevented this increase (Figs. 1G-I, 1Q). DHA also prevented RA-induced mitochondrial membrane depolarization; approximately $39.5 \%$ 

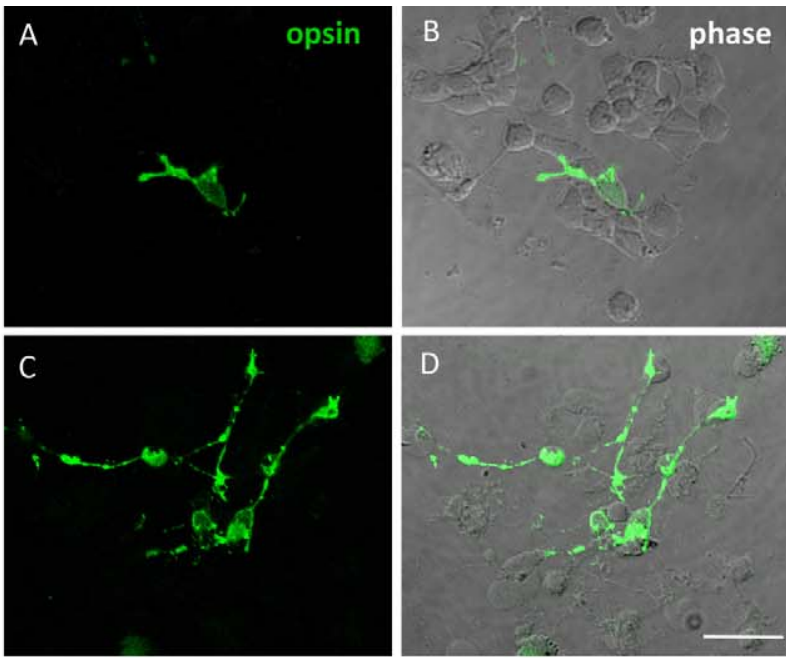

$\mathrm{E}$

Opsin expression

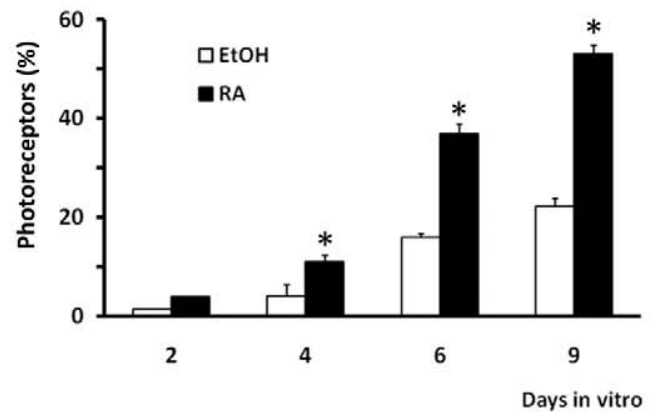

$\mathrm{F}$

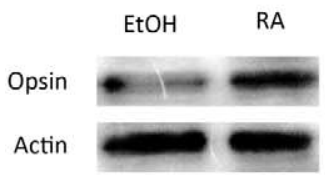

RA added at day 0
G

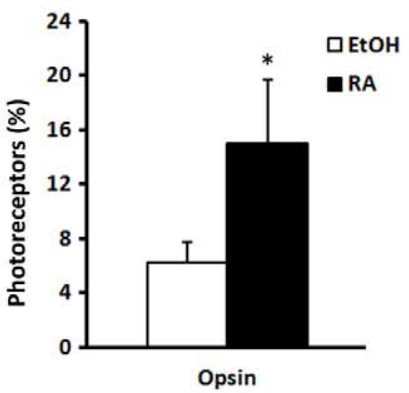

RA added at day 2

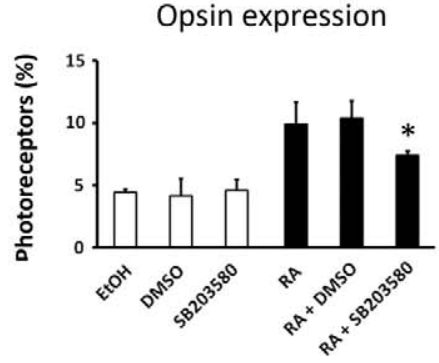

FigurE 5. Effect of RA on opsin expression. Cultures prepared from retinas from PN2 rats were supplemented with $\left(10^{-7}\right) \mathrm{M} \mathrm{RA}$, or with vehicle $(\mathrm{EtOH}) 1$ hour after plating. Fluorescence $(\mathbf{A}, \mathbf{C})$ and phase contrast (B, D) photomicrographs of 6-day old cultures show the increase in opsin expression (green) in RA-treated cultures $(\mathbf{C}, \mathbf{D})$ compared to controls (A, B). Changes in the percentages of opsinexpressing photoreceptors at different times of development are of photoreceptors preserved their mitochondrial potential in RA-treated cultures, whereas in those pretreated with DHA this value was $67.5 \%$, almost as in controls (Fig. 2G).

We then investigated whether DHA inhibited RA induction of apoptosis by blocking p38 activation. Pretreatment with DHA at day 0 did not preclude the increase in P-p38 levels induced by RA 1 to 4 hours after its addition (Fig. 3E). Hence, DHA protection of photoreceptors did not entail inactivation of p38 phosphorylation.

\section{RA Promoted the Simultaneous Activation of Apoptosis and Differentiation in Photoreceptors}

Since RA is known to promote photoreceptor differentiation, ${ }^{10,15,43-45}$ we investigated whether its addition at day 0 , which prompted apoptosis, simultaneously activated differentiation. Retina photoreceptors cultured in media lacking their trophic factors develop as round cells with a small cell body and a short cilium, ${ }^{3,4}$ and usually lack the high opsin levels and characteristic outer segments found in photoreceptors in vivo. Addition of RA at day 0 increased opsin expression (Figs. 5C, 5D), being most effective at $10^{-7} \mathrm{M}$ (not shown).

RA stimulation of opsin expression augmented during time in vitro. A small increase already was observed in RAsupplemented cultures at day 2 (Fig. 5E) and opsin-expressing photoreceptors increased from $4.1 \%$ to $11.1 \%$ at day 4 , from $16 \%$ to $37 \%$ at day 6 , and from $22.3 \%$ to nearly $53 \%$, at day 9 in controls and RA-supplemented cultures, respectively. This increase was confirmed by Western Blot (Fig. 5F).

RA increased the number of photoreceptors expressing peripherin/rds, a structural protein of the rims of outer segment discs, essential for their correct folding and maintenance (Figs. 6A-D). This protein accumulated in apical processes, structures that resemble the first steps of outer segment development (Figs. 6C, 6E, arrowheads). RA effect on peripherin expression was higher at early times in vitro; RA doubled the amount of cells expressing peripherin by day 4, whereas they increased $30 \%$ by day 6 (Fig. 6F). Western Blot analysis confirmed this increase (not shown). RA also induced the formation of peripherin-labeled cilia and apical processes (Figs. 6C-E, arrowheads). The number of cells that developed peripherin-positive cilia and apical processes increased from approximately 15,000 cells/dish in controls to nearly 22,000 cells/dish in RA-supplemented cultures (Fig. 6G). As a whole, these results support that addition of RA when photoreceptor progenitors still were cycling simultaneously induced their differentiation and apoptosis.

RA addition at day 2, when cell proliferation was over and RA no longer triggered apoptosis, increased opsin-positive photoreceptors from $6.2 \%$ in controls to $14.9 \%$ in RAsupplemented cultures at day 4 (Fig. 5G). This implies that RA enhanced photoreceptor differentiation independently of the presence of proliferating cells.

We then investigated whether inhibiting RA-induced apoptosis with Z-VAD-FMK increased photoreceptor differentiation after RA addition. RA doubled the percentage of opsin-

shown in (E). Western Blots show the increase in opsin levels in 6-day cultures treated with $10^{-7} \mathrm{M}$ RA at day 0 (F). The percentage of photoreceptors expressing opsin at day 4 in cultures treated without or with RA at day 2 is shown in (G); note that RA added at this time in vitro also increased opsin expression. Changes in the percentage of photoreceptors expressing opsin in control cultures; cultures treated at day 0 with RA; RA plus vehicle (DMSO) or the p38 inhibitor SB203580 are shown (H). Note that SB203580 only partially blocked RA effect on opsin expression. ${ }^{*} P<0.05$. Scale bar: $15 \mu \mathrm{m}$. 

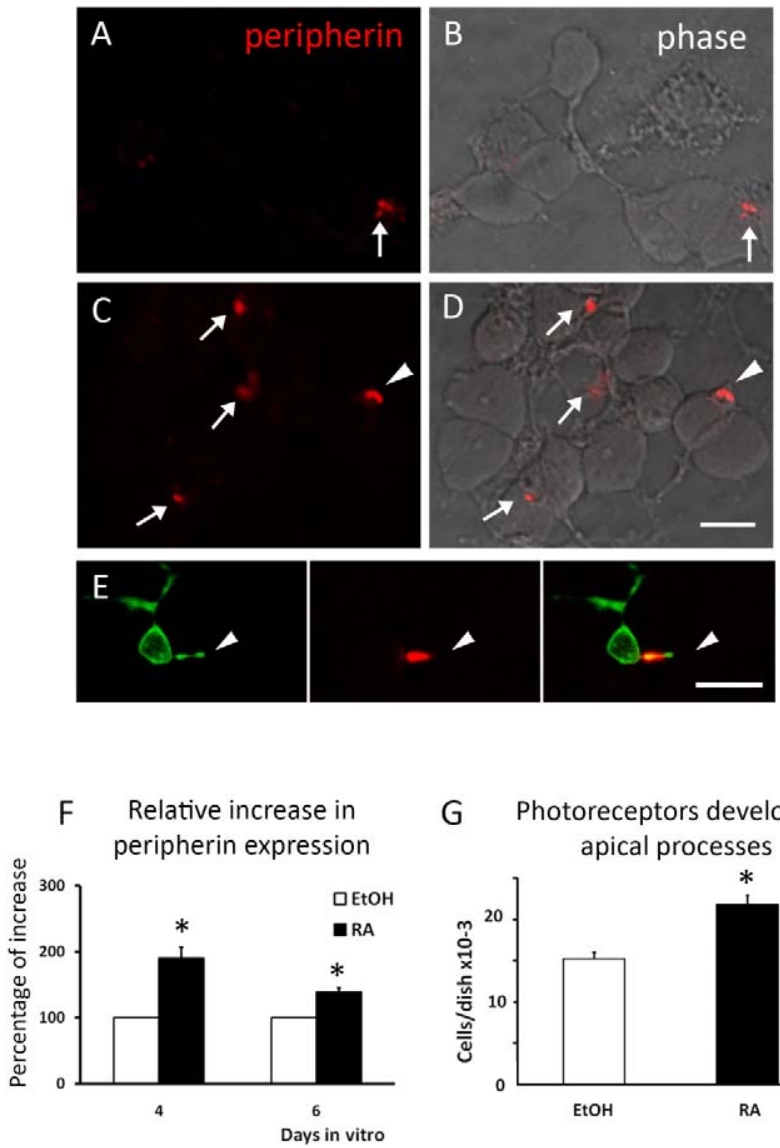

G Photoreceptors developing

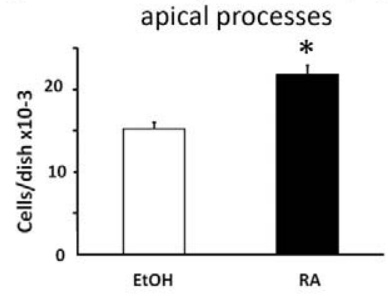

FigURE 6. Effect of RA on peripherin expression and formation of apical processes. Neuronal cultures were supplemented at day 0 without or with RA, and peripherin expression at days 4 and 6 was evaluated with an anti-peripherin monoclonal antibody. Phase contrast (B, D) and fluorescence (A, C, E) photomicrographs of 6-day cultures show that RA (C, D) increased the amount of photoreceptors expressing peripherin (arrows) compared to controls (A, B). The relative increase in peripherin expression in RA-supplemented cultures, compared to controls, is shown (F). RA also increased the amount of photoreceptors (G) that developed apical processes (arrowbeads in $[\mathbf{C}],[\mathbf{D}]$ and $[\mathbf{E}]$ ) compared to controls. ${ }^{*} P<0.05$. Scale bar: $15 \mu \mathrm{m}$.

positive photoreceptors, compared to controls cultures, but pretreatment with Z-VAD-FMK did not affect this increase (Table 2). This suggested that RA promoted the differentiation of a specific pool of photoreceptors and increasing photoreceptor survival did not augment this pool.

TABLE 2. Effect of RA-Induced Apoptosis Inhibition on Photoreceptor Differentiation

\begin{tabular}{lr}
\hline \multicolumn{1}{c}{ Treatment } & Opsin + \\
\hline EtOH & $5.9 \pm 1.3$ \\
ZVAD-FMK & $6.2 \pm 1.0$ \\
EtOH + ZVAD-FMK & $7.6 \pm 3.3$ \\
RA & $16.3 \pm 1.6$ \\
RA + ZVAD-FMK & $15.3 \pm 4.6$ \\
\hline
\end{tabular}

The percentage of opsin-positive photoreceptors at day 3 in vitro was determined in cultures treated with or without RA, and with or without the pan-caspase inhibitor ZVAD-FMK.
A

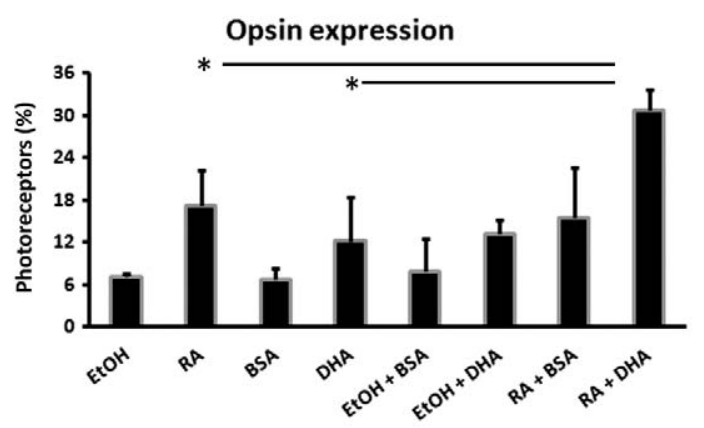

B

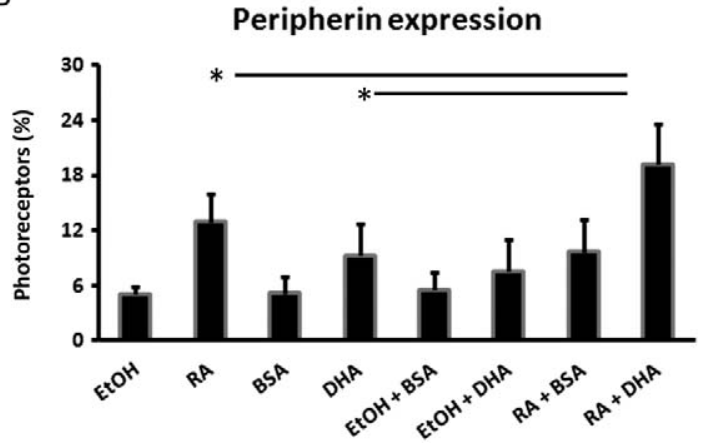

Figure 7. Additive effects of RA and DHA on photoreceptor differentiation. The percentages of photoreceptors expressing opsin (A) and peripherin (B) were analyzed in 6 day-cultures supplemented at day 0 with RA, DHA, their vehicles (EtOH and BSA, respectively), or RA plus DHA. While RA and DHA, added separately, increased the percentages of opsin (A) and peripherin (B) expressing photoreceptors, their combined addition induced a higher increase suggesting an additive effect of RA and DHA on photoreceptor differentiation. ${ }^{*} P<$ 0.05 .

\section{RA Effects on Opsin Expression Depended on p38 Activation}

We then evaluated whether p38 activation also was involved in RA effects on differentiation. Without SB203580, RA increased opsin-positive photoreceptors from approximately 5\% to $10.4 \%$ at day 6; addition of SB203580 at day 0 reduced RA enhancement of opsin expression (Fig. $5 \mathrm{H}$ ), but did not block it completely. This suggested that activation of p38 is one, but not the only, pathway involved in RA effect on differentiation.

\section{Additive Effects of DHA and RA on Photoreceptor Differentiation}

As DHA promotes photoreceptor differentiation, ${ }^{18}$ we explored whether its addition with RA had an additive or synergistic effect on this differentiation. At day 6 the percentage of opsin-positive cells increased from approximately $6 \%$ in controls to $16 \%$ and $12 \%$ in cultures with RA or DHA, respectively. Noteworthy, their combined addition led to an increment of approximately $30 \%$, which amounted to the sum of separate effects (Fig. 7A). RA and DHA showed a similar additive effect on peripherin expression (Fig. 7B).

\section{RA Promoted Neurite Outgrowth}

We then evaluated RA effect on neurite outgrowth. In controls, most photoreceptors developed short neurites (Figs. 8A-C), as 

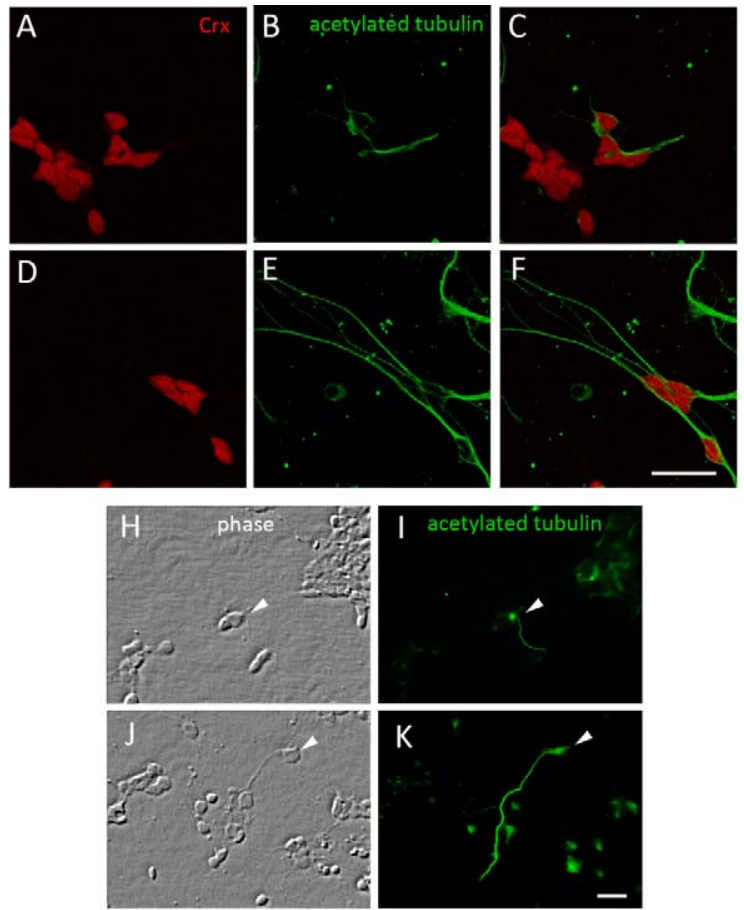
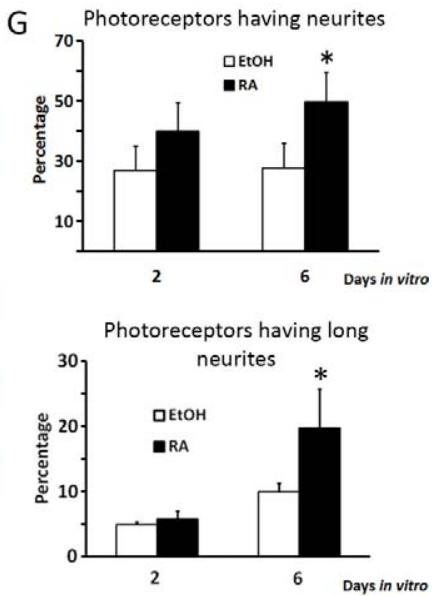

L Amacrine cells having long

neurites

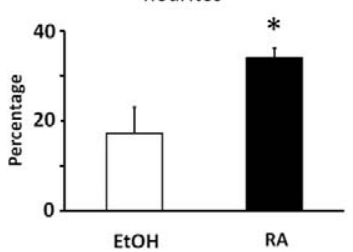

FigurE 8. Effect of RA on neurite outgrowth. Cultures prepared from PN2 pups were treated with RA at day 0, and neurite outgrowth was evaluated at days 2 and 6 , in photoreceptors, and 6 in amacrine cells. Fluorescence photomicrographs (A-F) of 6-day cultures show neurites in photoreceptors, identified by double-labeling with anti-CRX ([A, D], red) and anti-acetylated tubulin ([B, C, E, F], green) antibodies. RAsupplemented cultures (D-F) showed more photoreceptors having long neurites than controls (A-C). The percentage of photoreceptors having processes and of those having long processes ( $>3$ body diameters) in cultures without and with RA is shown (G). Phase contrast (H, J) and fluorescence (I, K) photomicrographs of 6-day cultures show amacrine cells had longer neurites in RA-treated cultures (J, K) than in controls (H, I). The percentage of amacrine cells having long processes $\left(>4\right.$ body diameters) is shown in $(\mathbf{L})$. ${ }^{*} P<0.05$. Scale bar: $15 \mu$ m.

evidenced by $\alpha$-acetylated tubulin labeling. RA significantly increased the sprouting and outgrowth of neurites; the number of photoreceptors having neurites (Fig. 8G) and bearing long neurites (i.e., $>3$ body diameters, Figs. 8D-F, 8G) was higher in RA-treated cultures than in controls. This effect was time dependent; while at day 2 RA had no effect, by day 6 the percentage of photoreceptors having long neurites increased from $9.9 \%$ in controls to $19.7 \%$, in RA-treated cultures (Fig. 8G). RA also promoted neurite outgrowth in amacrine cells (Figs. $8 \mathrm{H}-\mathrm{K})$. By day 6 , the percentage of these cells having long neurites (i.e., $>4$ body diameters) increased from $17.2 \%$ in controls to $34.1 \%$ in RA-treated cultures (Fig. 8L).

We then analyzed whether RA activated the p38 MAPK pathway to enhance neurite outgrowth. By day 6 , approxi-

Table 3. Effect of p38 Inhibition on Neurite Outgrowth

\begin{tabular}{lcc}
\hline \multicolumn{1}{c}{ Treatment } & $\begin{array}{c}\text { Amacrine Cells } \\
\text { Having Long } \\
\text { Neurites, } \%\end{array}$ & $\begin{array}{c}\text { Photoreceptors } \\
\text { Having Long } \\
\text { Neurites, \% }\end{array}$ \\
\hline EtOH & $14.2 \pm 0.9$ & $14.0 \pm 2.8$ \\
DMSO & $14.6 \pm 2.2$ & $15.2 \pm 3.1$ \\
SB203580 & $15.1 \pm 9.4$ & $13.0 \pm 1.7$ \\
RA & $29.3 \pm 1.6$ & $26.9 \pm 4.0$ \\
RA + SB203580 & $30.3 \pm 3.0$ & $29.4 \pm 7.1$ \\
\hline
\end{tabular}

Percentage of amacrine and photoreceptor neurons bearing long neurites ( $>4$ and 3 body diameters, respectively) in 6-day cultures treated at day 0 with RA, vehicle (EtOH), the p38 inhibitor (SB203580), its vehicle (DMSO), RA, or pretreated with SB203580 and 30 minutes later with RA (RA + SB203580, $N=3$ ). mately $30 \%$ of amacrine neurons and $27 \%$ of photoreceptors had long neurites in RA-supplemented cultures, and pretreatment with SB203580 before RA supplementation did not decrease these percentages (Table 3).

\section{RA Effect on Cell Cycle Exit}

We investigated whether RA advanced differentiation of photoreceptors by promoting an earlier exit of their progenitors from the cell cycle. Cultures from PNO retinas treated at day 2 with or without RA showed similar percentages of BrdUpositive cells (Table 4 ). Since by day 2 few photoreceptor progenitors remain in the cell cycle, we added RA at day 0 , when many progenitors still are proliferating ${ }^{46}$; this addition decreased slightly, though not significantly, the percentage of BrdU-labeled cells compared to controls (Table 4).

Similarly, RA decreased slightly, though not significantly, the percentage of cells expressing nestin, a neuroectoderm marker

TABLe 4. Effects of RA on Proliferation

\begin{tabular}{ccc}
\hline $\begin{array}{c}\text { Days } \\
\text { In Vitro }\end{array}$ & Treatment & BrdU/Total Cell $\boldsymbol{N}, \%$ \\
\hline \multirow{2}{*}{0} & EtOH & $17.5 \pm 4.6$ \\
& RA & $16.1 \pm 3.2$ \\
2 & EtOH & $2.9 \pm 2.5$ \\
& RA & $3.6 \pm 1.9$ \\
\hline
\end{tabular}

Retinal cell cultures prepared from PNO retinas were treated with vehicle (EtOH) or with RA at day 0 and pulse-labeled with BrdU for 4 hours before fixation after 12 or 48 hours in vitro. The percentage of BrdU-positive cells was determined by immunocytochemistry. 
Table 5. Effects of RA on Cell Cycle Progression

\begin{tabular}{clrcc}
\hline $\begin{array}{c}\text { Hours } \\
\text { In Vitro }\end{array}$ & Treatment & $\begin{array}{c}\text { Nestin }+/ \text { Total } \\
\text { Cell } \mathbf{N}, \%\end{array}$ & $\begin{array}{c}\text { p27 }+/ \text { Total } \\
\text { Cell } \mathbf{N}, \%\end{array}$ & $\begin{array}{c}\text { Pax6 }+/ \text { Total } \\
\text { Cell } \mathbf{N}, \%\end{array}$ \\
\hline \multirow{2}{*}{12} & EtOH & $32.5 \pm 6.2$ & $48.8 \pm 4.9$ & $13.6 \pm 2.2$ \\
& RA & $29.9 \pm 4.2$ & $43.9 \pm 4.8$ & $12.2 \pm 1.0$ \\
24 & EtOH & $15.2 \pm 3.5$ & $25.4 \pm 5.6$ & - \\
& RA & $12.3 \pm 3.1$ & $27.7 \pm 3.9$ & - \\
48 & EtOH & $3.3 \pm 1.2$ & $21.6 \pm 2.5$ & $6.8 \pm 2.5$ \\
& RA & $4.2 \pm 1.1$ & $25.3 \pm 4.3$ & $6.0 \pm 4.3$ \\
\hline
\end{tabular}

Retinal cell cultures were prepared from PNO and treated with vehicle (EtOH) or with RA at day 0 , as indicated in Table 4. The percentage of cells expressing p $27^{\mathrm{kip} 1}$ and the neuroectoderm marker nestin was determined after 12, 24, and 48 hours in vitro. The percentage of cells remaining in the cell cycle was established after 12 or 48 hours by determining the percentage of cells solely evidencing Pax6 expression at culture times; cells double labeled with Pax6 and HPC1, that is, amacrine neurons, were excluded.

present in proliferating cells, after 12 and 24 hours in culture. RA simultaneously induced a small, nonsignificant increase in cells expressing $\mathrm{p} 27^{\mathrm{kip} 1}$, a cell cycle inhibitor expressed by photoreceptors when exiting the cell cycle, compared to controls (Table 5).

Pax6 expression is present in all multipotent retinal progenitors ${ }^{27,29,47-49}$; it is lost in post mitotic photoreceptors, but it is preserved in amacrine cells. The percentage of cells that only expressed Pax6, excluding those double-labeled with HPC-1 and Pax6, that is, amacrine cells, decreased significantly between 12 and 48 hours after seeding the cells, reflecting the exit of photoreceptor progenitors from the cell cycle, ${ }^{27}$ and was unaffected by RA (Table 5). These results showed a tendency, but not a significant effect of RA on inducing an earlier cell cycle exit of photoreceptor progenitors.

We also investigated whether RA added at day 0 influenced cell fate or an early differentiation as photoreceptors of retinal progenitors. RA increased slightly, though not significantly, the percentage of cells expressing Crx, an early photoreceptor marker, compared to controls at day 1 and had no effect at day 2 (Table 6). The percentage of HPC-1-positive cells remained unchanged. This suggested that RA did not induce photoreceptor cell fate at this time in vitro.

\section{DisCuSSION}

RA is known to induce apoptosis and differentiation in diverse tissues, including the retina. ${ }^{10,11,50-52}$ We showed here that RA selectively promoted cell death and differentiation in retina photoreceptors. RA only induced an early onset of apoptosis when retinal progenitors still were in the cell cycle, whereas it advanced photoreceptor differentiation independently of the presence of proliferating progenitors. RA activation of the $\mathrm{p} 38$ MAPK signaling cascade was crucial for its induction of photoreceptor apoptosis; RA only activated this pathway at early developmental times and inhibiting this activation completely blocked its apoptotic effect. RA effect on photoreceptor differentiation also was dependent, though not exclusively, on the activation of the p38 MAPK pathway. Finally, our data showed that DHA, a trophic factor for photoreceptors, prevented RA-induced apoptosis and promoted an additive enhancement of differentiation in photoreceptors. A schematic summary of these findings is shown in Figure 9.

In the absence of trophic factors, photoreceptors start degenerating after 4 days in culture through an apoptotic pathway involving caspase activation and loss of mitochondrial membrane potential. ${ }^{4,38}$ We showed here that, if added while
Table 6. Effects of RA on Cell Fate Determination

\begin{tabular}{cllc}
\hline $\begin{array}{c}\text { Days } \\
\text { In Vitro }\end{array}$ & Marker & Treatment & $\begin{array}{c}\text { Marker+/ } \\
\text { Total Cell } \boldsymbol{N}, \%\end{array}$ \\
\hline \multirow{2}{*}{1} & Crx & EtOH & $63.5 \pm 2.0$ \\
& & RA & $66.8 \pm 0.7$ \\
& HPC1 & EtOH & $26.3 \pm 2.1$ \\
& & RA & $26.4 \pm 1.7$ \\
& Crx & EtOH & $69.1 \pm 3.4$ \\
& \multirow{2}{*}{ HPC1 } & RA & $69.4 \pm 6.8$ \\
& & EtOH & $25.4 \pm 3.1$ \\
& RA & $27.9 \pm 3.8$ \\
\hline
\end{tabular}

Retinal cell cultures were prepared from PNO retinas and treated with vehicle (EtOH) or with RA, as indicated in Table 4. The percentages of cells expressing Crx or HPC1 were determined by immunocytochemistry after 1 or 2 days in vitro.

photoreceptor progenitors still were active in the cell cycle, RA induced a premature cell death in photoreceptors through a similar apoptotic pathway, promoting mitochondrial depolarization and caspase activation. Noteworthy, RA addition when cells had exited the cell cycle no longer induced apoptosis (Fig. 9). RA has been shown to induce apoptosis in parallel with differentiation in undifferentiated cells, at a time that concurs with the onset of their neuronal phenotype, as occurs in the NT2 neuronal cell line. ${ }^{20}$ If RA forceful induction of differentiation in retinal progenitors when still immature is responsible of activating their apoptosis, we should expect RA to decrease proliferation and promote the early achievement of a photoreceptor cell fate as well. In rodents, many progenitor cells remain mitotically active until postnatal days 3 to 5 , and in neuronal cultures from PNO rat pups, these progenitors have their last mitotic divisions in vitro, with most of them adopting a photoreceptor fate by day $2 .^{27,46} \mathrm{RA}$ addition at day 0 neither decreased proliferation nor promoted an early onset of photoreceptor differentiation, as evidenced by the lack of increase in Crx expression. This suggests that an early exit from the cell cycle is not the main cause of RA-induced apoptosis.

A clue to the differential effect of RA on apoptosis with time in vitro might reside in its ability to activate the p38 MAPK signaling pathway. This pathway has been associated with stress responses and linked to regulation of apoptosis induced by several insults in different systems. ${ }^{32-37}$ The p38 family includes four known members $\left(\mathrm{p} 38_{\alpha}, \mathrm{p} 38_{\beta}, \mathrm{p} 38_{\gamma}\right.$, and $\left.\mathrm{p} 38_{\delta}\right)$, all of which share significant identity with each other. Members of the p38 family exhibit serine-kinase activities that, in turn, activate or regulate other serine kinases. In the retina, p38 activation leads to oxidative stress-induced apoptosis of a retinal pigment epithelium cell line and to light-induced apoptosis of the $661 \mathrm{~W}$ cone-like cell line. ${ }^{53-55} \mathrm{RA}$ effects in vertebrates are mediated by its binding and activating two families of nuclear receptors, RAR and RXR. These nuclear receptors are ligand-dependent transcriptional regulators, which induce gene transcription after binding to RA response elements present in the promoters of RA-inducible genes. ${ }^{56,57}$ RARs are present in the developing neural retina, ${ }^{58}$ and activation of the p38 MAPK in response to RA is involved in the turnover of some RAR subunits and in RA-induced transactivation activity. ${ }^{32,59,60}$ Our results demonstrated that RA activated the p38 MAPK pathway in retina photoreceptors at early times in culture (Fig. 9). Activation of p38 was crucial for the pro-apoptotic effect of RA, since inhibiting this activation completely prevented RA-induced apoptosis in photoreceptors. Moreover, when added at day 2, RA was unable to increase P-p38 levels and, therefore, no longer induced photoreceptor apoptosis. RA did not affect the levels 


\section{Progenitor}

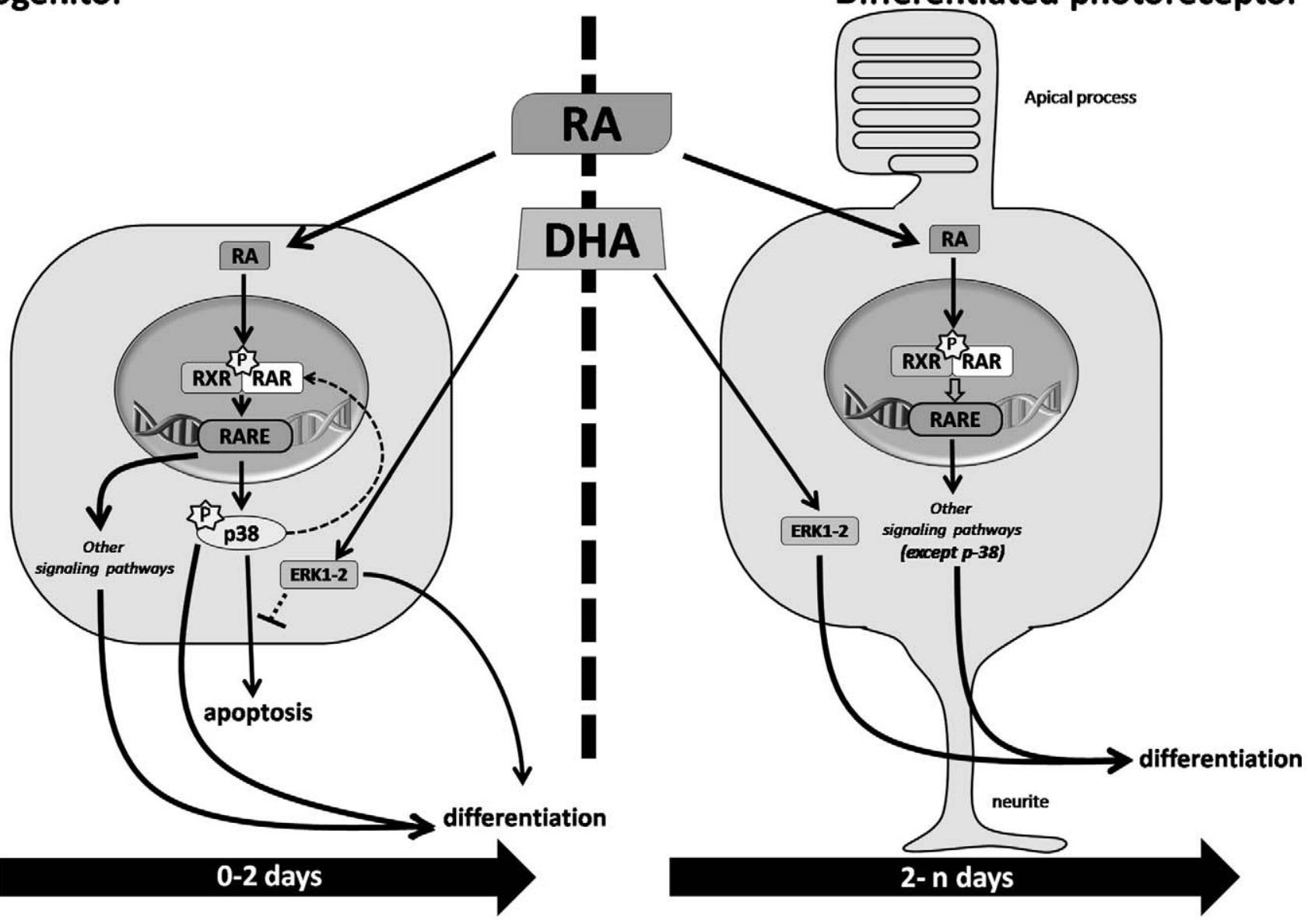

FIGURE 9. Mechanisms of RA effects on retina photoreceptors. During early stages of retina development (left panel), when retina progenitors still were progressing in the cell cycle, RA bound and activated its nuclear receptors (RAR and RXR) to activate the p38 MAPK signaling pathway. Activation of this pathway was crucial for induction of apoptosis and also involved in promoting photoreceptor differentiation, together with other signaling cascades, which are likely to be triggered by RAR/RXR activation. DHA, a photoreceptor trophic factor, prevented cell death probably by activating the ERK/MAPK pathway, ${ }^{38}$ which blocked the apoptotic signal downstream of $\mathrm{p} 38$ activation. In post mitotic photoreceptors (right panel) RA lost its capacity to activate the p38 pathway and no longer induced apoptosis; however, it still promoted differentiation, most likely by acting through other, still undefined, signaling pathways.

of phosphorylated ERK, implying that its induction of apoptosis did not involve modulation of the ERK/MAPK. These results suggested that at early developmental stages RA triggers apoptosis in photoreceptors through the activation of the p38 MAPK pathway; developmental changes lead to the loss of RA capacity to activate this pathway and, consequently, to the loss of its pro-apoptotic effect (Fig. 9).

The finding that RA did not induce the apoptosis of amacrine cells supports the hypothesis that activation of the p38 MAPK pathway is essential for its pro-apoptotic effect. Activation of this pathway, as evidenced by an increase in Pp38 levels, was observed chiefly in photoreceptors, suggesting it either was absent or not responsive to RA in amacrine neurons. This implies that different signals and signaling cascades are involved in the decision between death and survival in amacrine and photoreceptor neurons.

DHA, which rescues photoreceptors from apoptosis during their development in vitro, ${ }^{4,61}$ also prevented the untimely photoreceptor death induced by RA. DHA protection involves the activation of the ERK/MAPK survival pathway in photoreceptors $^{38}$ and our data suggested that this pathway was not regulated by RA. DHA protection did not involve blocking RA activation of the p38 pathway, since P-p38 levels were similar in cultures treated with RA in the presence or absence of DHA.
Moreover, DHA protected photoreceptors from apoptosis in spite of RA activation of the $\mathrm{p} 38$ pathway. RA has been shown to induce cell death by phosphorylating and modulating the activity of $\mathrm{Bim}_{\mathrm{EL}}$, a member of the $\mathrm{Bcl}-2$ family ${ }^{62}$; however, phosphorylation of $\operatorname{Bim}_{\mathrm{EL}}$ by ERK1/2 at additional sites antagonizes apoptosis. ${ }^{63-65}$ Hence, through activation of the ERK/MAPK pathway DHA might have a crucial role in blocking RA-induced apoptosis in photoreceptors (Fig. 9).

In vivo, development of photoreceptors is a multistep process; once they exit the cell cycle, photoreceptors start to express specific proteins and develop a connecting cilium, at the tip of which opsin-enriched membranes are assembled. In vitro, the differentiation of photoreceptors seems to be arrested in the absence of trophic factors, with photoreceptors resembling their immature in vivo counterparts ${ }^{27}$; few of them express opsin and peripherin and even fewer develop apical processes. RA regulates different steps in photoreceptor development. It increases differentiation in chick photoreceptors, ${ }^{13}$ accelerates the onset of opsin expression in cultured rat retina explants and retina neurons, ${ }^{16,17}$ and is required for the proper organization of nascent outer segment membranes in Xenopus laevis tadpoles. ${ }^{66}$ Our results showed that RA rapidly advanced photoreceptor differentiation in culture. RA promoted an early onset of opsin expression, increased the levels of 
peripherin, and stimulated the formation of apical processes. RA also promoted neurite outgrowth in photoreceptors. RA has a crucial role in the induction of rod photoreceptor cell fate, in embryonic retinas from rat and zebrafish, ${ }^{10,15,44}$ in retinal progenitor cells derived from mouse and human stem cells, and in Müller glia-derived retinal progenitors. ${ }^{67,68}$ During retina neurogenesis in zebrafish, RA influences a rod versus cone photoreceptor cell fate, ${ }^{69}$ and it induces retinal progenitors to acquire a photoreceptor cell fate in E15 and E18 rat embryos. ${ }^{10}$ Since RA did not affect photoreceptor cell fate in retinal progenitors, our data supported that the higher number of cells exhibiting opsin and peripherin expression in RAsupplemented cultures resulted from an early activation of their development as photoreceptors.

In contrast with its effect on apoptosis, RA enhancement of photoreceptor differentiation was independent of the presence of proliferating cells. Inhibiting the p38 MAPK pathway partially blocked RA effect on differentiation. Since this pathway is activated only at early stages of photoreceptor development, these data suggested that the p38 signaling pathway is a relevant, though not the only pathway involved in stimulating photoreceptors differentiation. Thus, RA would activate the selective transcription of genes involved in photoreceptor differentiation through different pathways at different stages of development (Fig. 9). Photoreceptor trophic factors, such as FGFb and DHA, also activate the ERK/MAPK signaling cascade ${ }^{38}$ to promote opsin expression. Interestingly, the combined addition of RA and DHA to the cultures led to an additive effect on the amount of opsin-expressing photoreceptors. These results imply that RA and DHA activate independent pathways to advance photoreceptor differentiation.

Blocking apoptosis with a caspase inhibitor did not increase the amount of differentiated photoreceptors, as might be expected from increasing the pool of viable photoreceptors. This suggested that RA stimulation of differentiation and its triggering of apoptosis affect specific photoreceptor pools. Alternatively, photoreceptors that were meant to die through an apoptotic pathway might be arrested in their development at a stage that makes them unable to initiate their differentiation, even if caspase-mediated cell death is interrupted.

RA stimulation of neurite sprouting and outgrowth in photoreceptors and amacrine cells also might involve activation of RAR, since retinoids have been shown to activate RAR $_{\beta 2}$ to promote neurite outgrowth of cultured embryonic dorsal root ganglia (DRG), and spinal cord and adult DRG. ${ }^{70-72}$ Inhibition of $\mathrm{p} 38$ MAPK did not prevent this stimulation, suggesting that RA activated other intracellular pathways to promote neurite elongation. Stimulation of neurite outgrowth was the only observed effect of RA in amacrine neurons, and lack of involvement of the p38 MAPK pathway in this effect is consistent with the evidence that RA only activated p38 in photoreceptors. Activation of insulin receptors has been shown to promote neurite outgrowth in photoreceptors and amacrine neurons, ${ }^{26,73}$ the PI3K signaling pathway. ${ }^{74}$ Establishing whether RA activates this pathway to promote neurite outgrowth in these cells requires further research.

Development of photoreceptors depends on many factors, with RA having a crucial role. While approximately $50 \%$ of amacrine neurons disappear during retina development, only $5 \%$ of the initially generated photoreceptors die. ${ }^{31}$ This implies that a precise synchronization in the supply of the molecular signals essential for photoreceptor development is crucial; an untimely provision of RA in the absence of survival molecules might activate apoptosis. Our results underscore the relevance of a fine balance between the molecules and mechanisms regulating genesis and differentiation of photoreceptors, and those promoting their survival to achieve the precise number of photoreceptors for adequate visual function.

\section{Acknowledgments}

Luis E. Politi and Nicole P. Rotstein are principal and independent researchers from the Argentinean National Research Council (CONICET), and Professors of Cell Biology and Biological Chemistry, respectively, at the Universidad Nacional del Sur, Bahía Blanca, Argentina. Pablo De Genaro and M. Victoria Simón have CONICET Doctoral and postdoctoral fellowships, respectively.

Supported by grants from FONCyT (PICT 2006-711), the Argentinean National Research Council (CONICET), and the Universidad Nacional del Sur, Bahía Blanca, Argentina.

Disclosure: P. De Genaro, None; M.V. Simón, None; N.P. Rotstein, None; L.E. Politi, None

\section{References}

1. Jacobson MD, Weil M, Raff MC. Programmed cell death in animal development. Cell. 1997;88:347-354.

2. Raff MC, Barres BA, Burne JF, Coles HS, Ishizaki Y, Jacobson MD. Programmed cell death and the control of cell survival: lessons from the nervous system. Science. 1993;262:695-700.

3. Rotstein NP, Aveldano MI, Barrantes FJ, Politi LE. Docosahexaenoic acid is required for the survival of rat retinal photoreceptors in vitro. J Neurochem. 1996;66:1851-1859.

4. Rotstein NP, Aveldano MI, Barrantes FJ, Roccamo AM, Politi LE. Apoptosis of retinal photoreceptors during development in vitro: protective effect of docosahexaenoic acid. J Neurochem. 1997;69:504-513.

5. LaVail MM, Yasumura D, Matthes MT, et al. Protection of mouse photoreceptors by survival factors in retinal degenerations. Invest Ophthalmol Vis Sci. 1998;39:592-602.

6. Fontaine V, Kinkl N, Sahel J, Dreyfus H, Hicks D. Survival of purified rat photoreceptors in vitro is stimulated directly by fibroblast growth factor-2. J Neurosci. 1998;18:9662-9672.

7. Frasson M, Picaud S, Leveillard T, et al. Glial cell line-derived neurotrophic factor induces histologic and functional protection of rod photoreceptors in the $\mathrm{rd} / \mathrm{rd}$ mouse. Invest Ophthalmol Vis Sci. 1999;40:2724-2734.

8. Politi LE, Rotstein NP, Carri NG. Effect of GDNF on neuroblast proliferation and photoreceptor survival: additive protection with docosahexaenoic acid. Invest Ophthalmol Vis Sci. 2001; 42:3008-3015.

9. Altschuler LR, Parisi MN, Cageao LF, Chiocchio SR, FernandezPol JA, Zaninovich AA. Epidermal growth factor stimulates thyrotropin secretion in the rat. Neuroendocrinology. 1993; 57:23-27.

10. Kelley MW, Turner JK, Reh TA. Retinoic acid promotes differentiation of photoreceptors in vitro. Development. 1994;120:2091-2102.

11. Sporn MB, Roberts $\mathrm{AB}$. Role of retinoids in differentiation and carcinogenesis. J Natl Cancer Inst. 1984;73:1381-1387.

12. Apfel C, Bauer F, Crettaz M, et al. A retinoic acid receptor alpha antagonist selectively counteracts retinoic acid effects. Proc Natl Acad Sci U S A. 1992;89:7129-7133.

13. Stenkamp DL, Gregory JK, Adler R. Retinoid effects in purified cultures of chick embryo retina neurons and photoreceptors. Invest Ophthalmol Vis Sci. 1993;34:2425-2436.

14. Barritault D, Arruti C, Courtois Y. Is there a ubiquitous growth factor in the eye? Proliferation induced in different cell types by eye-derived growth factors. Differentiation. 1981;18:2942.

15. Kelley MW, Williams RC, Turner JK, Creech-Kraft JM, Reh TA. Retinoic acid promotes rod photoreceptor differentiation in rat retina in vivo. Neuroreport. 1999;10:2389-2394.

16. Wallace VA, Jensen AM. IBMX, taurine and 9-cis retinoic acid all act to accelerate rhodopsin expression in postmitotic cells. Exp Eye Res. 1999;69:617-627. 
17. Soderpalm AK, Fox DA, Karlsson JO, van Veen T. Retinoic acid produces rod photoreceptor selective apoptosis in developing mammalian retina. Invest Ophthalmol Vis Sci. 2000;41:937947.

18. Rotstein NP, Politi LE, Aveldano MI. Docosahexaenoic acid promotes differentiation of developing photoreceptors in culture. Invest Ophthalmol Vis Sci. 1998;39:2750-2758.

19. Patel NA, Song SS, Cooper DR. PKCdelta alternatively spliced isoforms modulate cellular apoptosis in retinoic acid-induced differentiation of human NT2 cells and mouse embryonic stem cells. Gene Expr. 2006;13:73-84.

20. Guillemain I, Fontes G, Privat A, Chaudieu I. Early programmed cell death in human NT2 cell cultures during differentiation induced by all-trans-retinoic acid. J Neurosci Res. 2003;71:38-45.

21. Politi LE, Lehar M, Adler R. Development of neonatal mouse retinal neurons and photoreceptors in low density cell culture. Invest Ophthalmol Vis Sci. 1988;29:534-543.

22. Adler R. Regulation of neurite growth in purified retina neuronal cultures: effects of PNPF, a substratum-bound, neurite-promoting factor. J Neurosci Res. 1982;8:165-177.

23. Barnstable CJ. Monoclonal antibodies which recognize different cell types in the rat retina. Nature. 1980;286:231-235.

24. Hicks D, Barnstable CJ. Different rhodopsin monoclonal antibodies reveal different binding patterns on developing and adult rat retina. J Histochem Cytochem. 1987;35:13171328.

25. Kljavin IJ, Lagenaur C, Bixby JL, Reh TA. Cell adhesion molecules regulating neurite growth from amacrine and rod photoreceptor cells. J Neurosci. 1994;14:5035-5049.

26. Politi LE, Rotstein NP, Salvador G, Giusto NM, Insua MF. Insulin-like growth factor-I is a potential trophic factor for amacrine cells. $J$ Neurochem. 2001;76:1199-1211.

27. Garelli A, Rotstein NP, Politi LE. Docosahexaenoic acid promotes photoreceptor differentiation without altering Crx expression. Invest Ophthalmol Vis Sci. 2006;47:3017-3027.

28. Abrahan CE, Miranda GE, Agnolazza DL, Politi LE, Rotstein NP. Synthesis of sphingosine is essential for oxidative stressinduced apoptosis of photoreceptors. Invest Ophthalmol Vis Sci. 2010;51:1171-1180.

29. Simon MV, De Genaro P, Abrahan CE, de Los SB, Rotstein NP, Politi LE. Muller glial cells induce stem cell properties in retinal progenitors in vitro and promote their further differentiation into photoreceptors. J Neurosci Res. 2011;90: 407-421.

30. Laemmli UK, Beguin F, Gujer-Kellenberger G. A factor preventing the major head protein of bacteriophage $\mathrm{T} 4$ from random aggregation. J Mol Biol. 1970;47:69-85.

31. Cepko CL, Austin CP, Yang X, Alexiades M, Ezzeddine D. Cell fate determination in the vertebrate retina. Proc Natl Acad Sci U S A. 1996;93:589-595.

32. Alsayed Y, Uddin S, Mahmud N, et al. Activation of Rac1 and the p38 mitogen-activated protein kinase pathway in response to all-trans-retinoic acid. J Biol Chem. 2001;276:4012-4019.

33. Ichijo $\mathrm{H}$, Nishida $\mathrm{E}$, Irie $\mathrm{K}$, et al. Induction of apoptosis by ASK1, a mammalian MAPKKK that activates SAPK/JNK and p38 signaling pathways. Science. 1997;275:90-94.

34. Kummer JL, Rao PK, Heidenreich KA. Apoptosis induced by withdrawal of trophic factors is mediated by p38 mitogenactivated protein kinase. J Biol Chem. 1997;272:2049020494.

35. Miyazawa K, Mori A, Miyata H, Akahane M, Ajisawa Y, Okudaira H. Regulation of interleukin-1beta-induced interleukin-6 gene expression in human fibroblast-like synoviocytes by p38 mitogen-activated protein kinase. J Biol Chem. 1998;273: 24832-24838.
36. Shapiro L, Dinarello CA. Osmotic regulation of cytokine synthesis in vitro. Proc Natl Acad Sci U S A. 1995;92:1223012234.

37. Xia Z, Dickens M, Raingeaud J, Davis RJ, Greenberg ME. Opposing effects of ERK and JNK-p38 MAP kinases on apoptosis. Science. 1995;270:1326-1331.

38. German OL, Insua MF, Gentili C, Rotstein NP, Politi LE. Docosahexaenoic acid prevents apoptosis of retina photoreceptors by activating the ERK/MAPK pathway. $J$ Neurochem. 2006;98:1507-1520.

39. La Vail MM, Rapaport DH, Rakic P. Cytogenesis in the monkey retina. J Comp Neurol. 1991;309:86-114.

40. Miranda GE, Abrahan CE, Politi LE, Rotstein NP. Sphingosine-1phosphate is a key regulator of proliferation and differentiation in retina photoreceptors. Invest Ophthalmol Vis Sci. 2009;50:4416-4428.

41. Ogilvie JM, Speck JD, Lett JM. Growth factors in combination, but not individually, rescue rd mouse photoreceptors in organ culture. Exp Neurol. 2000;161:676-685.

42. Rotstein NP, Politi LE, German OL, Girotti R. Protective effect of docosahexaenoic acid on oxidative stress-induced apoptosis of retina photoreceptors. Invest Ophthalmol Vis Sci. 2003;44: 2252-2259.

43. Marsh-Armstrong N, McCaffery P, Gilbert W, Dowling JE, Drager UC. Retinoic acid is necessary for development of the ventral retina in zebrafish. Proc Natl Acad Sci U S A. 1994;91:7286-7290.

44. Hyatt GA, Schmitt EA, Fadool JM, Dowling JE. Retinoic acid alters photoreceptor development in vivo. Proc Natl Acad Sci U S A. 1996;93:13298-13303.

45. Khanna H, Akimoto M, Siffroi-Fernandez S, Friedman JS, Hicks $\mathrm{D}$, Swaroop A. Retinoic acid regulates the expression of photoreceptor transcription factor NRL. J Biol Chem. 2006; 281:27327-27334.

46. Insua MF, Garelli A, Rotstein NP, German OL, Arias A, Politi LE. Cell cycle regulation in retinal progenitors by glia-derived neurotrophic factor and docosahexaenoic acid. Invest $O p h$ thalmol Vis Sci. 2003;44:2235-2244.

47. Marquardt T, Ashery-Padan R, Andrejewski N, Scardigli R, Guillemot F, Gruss P. Pax6 is required for the multipotent state of retinal progenitor cells. Cell. 2001;105:43-55.

48. Marquardt T, Gruss P. Generating neuronal diversity in the retina: one for nearly all. Trends Neurosci. 2002;25:32-38.

49. Morrow EM, Belliveau MJ, Cepko CL. Two phases of rod photoreceptor differentiation during rat retinal development. $J$ Neurosci. 1998;18:3738-3748.

50. Sporn MB, Roberts AB, Goodman D. The Retinoids: Biology, Chemistry, and Medicine. New York: Raven Press; 1994.

51. Prabhudesai SN, Cameron DA, Stenkamp DL. Targeted effects of retinoic acid signaling upon photoreceptor development in zebrafish. Dev Biol. 2005;287:157-167.

52. Hyatt GA, Dowling JE. Retinoic acid. A key molecule for eye and photoreceptor development. Invest Ophthalmol Vis Sci. 1997;38:1471-1475.

53. Ho TC, Yang YC, Cheng HC, et al. Activation of mitogenactivated protein kinases is essential for hydrogen peroxideinduced apoptosis in retinal pigment epithelial cells. Apoptosis. 2006;11:1899-1908.

54. Yang LP, Zhu XA, Tso MO. Role of NF-kappaB and MAPKs in light-induced photoreceptor apoptosis. Invest Ophthalmol Vis Sci. 2007;48:4766-4776.

55. Yang LP, Zhu XA, Tso MO. A possible mechanism of microgliaphotoreceptor crosstalk. Mol Vis. 2007;13:2048-2057.

56. Chambon $P$. The retinoid signaling pathway: molecular and genetic analyses. Semin Cell Biol. 1994;5:115-125.

57. Kastner P, Mark M, Chambon P. Nonsteroid nuclear receptors: what are genetic studies telling us about their role in real life? Cell. 1995;83:859-869. 
58. De Leeuw AM, Gaur VP, Saari JC, Milam AH. Immunolocalization of cellular retinol-, retinaldehyde- and retinoic acidbinding proteins in rat retina during pre- and postnatal development. J Neurocytol. 1990;19:253-264.

59. Gianni M, Kopf E, Bastien J, et al. Down-regulation of the phosphatidylinositol 3-kinase/Akt pathway is involved in retinoic acid-induced phosphorylation, degradation, and transcriptional activity of retinoic acid receptor gamma $2 . J$ Biol Chem. 2002;277:24859-24862.

60. Gianni M, Bauer A, Garattini E, Chambon P, Rochette-Egly C. Phosphorylation by p38MAPK and recruitment of SUG-1 are required for RA-induced RAR gamma degradation and transactivation. EMBO J. 2002;21:3760-3769.

61. German OL, Buzzi E, Rotstein NP, Rodriguez-Boulan E, Politi LE. Retinal pigment epithelial cells promote spatial reorganization and differentiation of retina photoreceptors. J Neurosci Res. 2008;86:3503-3514.

62. Cai B, Chang SH, Becker EB, Bonni A, Xia Z. p38 MAP kinase mediates apoptosis through phosphorylation of BimEL at Ser65. J Biol Chem. 2006;281:25215-25222.

63. Biswas SC, Greene LA. Nerve growth factor (NGF) downregulates the $\mathrm{Bcl}-2$ homology $3(\mathrm{BH} 3)$ domain-only protein Bim and suppresses its proapoptotic activity by phosphorylation. J Biol Chem. 2002;277:49511-49516.

64. Harada H, Quearry B, Ruiz-Vela A, Korsmeyer SJ. Survival factor-induced extracellular signal-regulated kinase phosphorylates BIM, inhibiting its association with BAX and proapoptotic activity. Proc Natl Acad Sci U S A. 2004;101:1531315317.

65. Ley R, Ewings KE, Hadfield K, Howes E, Balmanno K, Cook SJ. Extracellular signal-regulated kinases $1 / 2$ are serum-stimulated "Bim(EL) kinases" that bind to the BH3-only protein Bim(EL) causing its phosphorylation and turnover. J Biol Chem. 2004; 279:8837-8847.
66. Wang X, Tong Y, Giorgianni F, Beranova-Giorgianni S, Penn JS, Jablonski MM. Cellular retinol binding protein 1 modulates photoreceptor outer segment folding in the isolated eye. Dev Neurobiol. 2010;70:623-635.

67. Osakada F, Ooto S, Akagi T, Mandai M, Akaike A, Takahashi M. Wnt signaling promotes regeneration in the retina of adult mammals. J Neurosci. 2007;27:4210-4219.

68. Osakada F, Ikeda H, Sasai Y, Takahashi M. Stepwise differentiation of pluripotent stem cells into retinal cells. Nat Protoc. 2009; $4: 811-824$.

69. Stevens CB, Cameron DA, Stenkamp DL. Plasticity of photoreceptor-generating retinal progenitors revealed by prolonged retinoic acid exposure. BMC Dev Biol. 2011;11:51.

70. Corcoran J, Shroot B, Pizzey J, Maden M. The role of retinoic acid receptors in neurite outgrowth from different populations of embryonic mouse dorsal root ganglia. J Cell Sci. 2000; 113(Pt 14):2567-2574.

71. Corcoran J, So PL, Barber RD, et al. Retinoic acid receptor beta2 and neurite outgrowth in the adult mouse spinal cord in vitro. J Cell Sci. 2002;115:3779-3786.

72. So PL, Yip PK, Bunting S, et al. Interactions between retinoic acid, nerve growth factor and sonic hedgehog signalling pathways in neurite outgrowth. Dev Biol. 2006;298:167175.

73. Rajala RV, Rajala A, Brush RS, Rotstein NP, Politi LE. Insulin receptor signaling regulates actin cytoskeletal organization in developing photoreceptors. J Neurochem. 2009;110:16481660.

74. Li G, Rajala A, Wiechmann AF, Anderson RE, Rajala RV. Activation and membrane binding of retinal protein kinase Balpha/Akt1 is regulated through light-dependent generation of phosphoinositides. J Neurochem. 2008;107:1382-1397. 